\title{
Interactive aspects of producers and waste-disposal firms out of a market boundary
}

\author{
Eiji Hosoda*
}

${ }^{*}$ Correspondence:

hosoda@econ.keio.ac.jp

Faculty of Economics, Keio University, 2-15-45 Mita,

Minato-ku, Tokyo 108-8345 Japan

\section{Springer}

\begin{abstract}
This paper examines how environmentally friendly production and disposal of waste are promoted by interaction between producers and disposal firms out of a market boundary. Utilizing the dual nature of price and quantity systems, I show that the concept of natural price or production price can be applied to constructing a sustainable economy, where production and waste disposal are made with a relatively small environmental burden. I demonstrate that such a sustainable economy can be created by extended producer responsibility.
\end{abstract}

Keywords: Waste, Reproducibility, Sustainable equilibrium , Extended producer responsibility

JEL Classification: E11, O41, Q53

\section{Background}

Understanding of inter-industrial relationships is indispensable for solving environmental problems. Without knowing how industries are interconnected with one another, no one should be able to grasp how an environmental restriction imposed on an industry affects other industries directly or indirectly and what the final result would be. A waste problem is no exception. A requirement for reduction of waste generated from an endof-life product (ELP) should affect not only the industry which produces that product but also other industries indirectly.

When it comes to a waste problem, however, there is a peculiar feature on an interactive aspect between industries, interaction between environmentally friendly producers and environmentally friendly waste-disposal firms out of a market boundary. It is often found that producers and waste-disposal firms are interactively or cooperatively working, in a certain institutional environment, for waste reduction as well as prevention of pollution which might be accompanied with waste treatment. Such an interactive nature has attracted a great deal of attention, since it is considered to promote the so-called design for environment (DfE) and contribute to controlling the flow of environmental burdens at the production stage.

Yet, to the best of the author's knowledge, this problem has not been dealt with satisfaction by researchers so far. The purpose of this paper is to address this problem by

(c) 2016 Hosoda. This article is distributed under the terms of the Creative Commons Attribution 4.0 International License (http:// creativecommons.org/licenses/by/4.0/), which permits unrestricted use, distribution, and reproduction in any medium, provided you give appropriate credit to the original author(s) and the source, provide a link to the Creative Commons license, and indicate if changes were made. 
means of a Sraffian type of inter-industrial reproduction model based upon Sraffa (1960), which is supposed to be most suitable to the analysis of the problem. ${ }^{1}$ By so doing, I demonstrate how environmentally friendly production and waste disposal are successfully implemented in an economy and, in this sense, how a sustainable economy is realized. It will be shown that such implementation can be interpreted as an application of extended producer responsibility (EPR), which is proposed by OECD.

Although problems of waste disposal or recycling have not been taken up by Sraffian economists until quite recently, rudimentary works can be found in the rather early stages of the research. Steedman $(1975,1977)$ shows that negative value plays a crucial role in the analysis of positive profits with negative surplus value. Certainly, he does not explicitly deal with waste problems in his works, but his anatomy of negative value potentially has the seed of the study of waste problems: The idea of negative value can naturally be developed to that of negative prices, and those prices can be interpreted as a scarcity index of bads or dis-commodities.

Franke's work (1986) shows that negative prices cannot be avoided in a joint production model, when a free-disposal assumption is abandoned so that bads or dis-commodities are taken into account. It may be said that his work is a starting point of the analysis of waste problems by means of a Sraffa model, although it is so general that concrete issues on waste treatment are not discussed explicitly.

Fujimoto and Opocher (2010), Lager (2001) and Hosoda (2010b) are more explicit in dealing with bads or dis-commodities in a Sarrafian type of model. Taking residuals discharged from economic activities into account and discarding a free-disposal assumption, they demonstrate how a capitalist economy can reproduce itself in the long run. Clearly, a costly disposal process, whether it is a waste-disposal process or a recycling process, is crucial for the reproducibility of an economy.

Although those works have contributed to the development of the Sraffian analysis toward exploration of relationships between an economy and environment, they more or less remain at an abstract level, having a distance from applicability to real environmental policies. Circumstances have, however, changed gradually. Hosoda (2010a) utilizes a Sraffian type of model to demonstrate the necessity of EPR, which is an effective waste management policy and has been adopted in many countries. He also applies the model to a realistic situation in which a secondary material obtained by recycling is degraded, demonstrating that the conventional Sraffian model can be naturally extended to the one which describes the transaction of commodities and bads or dis-commodities in a modern recycling society (Hosoda 2012, 2014). ${ }^{2}$

The present work is situated in a natural extension of the research line of the above studies and shares the basic structure and characteristics with them. I would like, however, to emphasize that there is a big difference between the present work and the previous ones: The important feature that production processes and waste-disposal processes are interconnected through the effects which are given outside market transactions is fully taken into account and analyzed. Environmentally friendly production, represented

\footnotetext{
${ }^{1}$ There are also some similarities between the basic model of this paper and a von Neumann model. Refer to Kurz and Salvadori (1995) on those similarities.

2 See also Gehrke and Lager (1995) and Lager (1999) as examples of interesting application of a Sraffian model to environmental problems.
} 
by, say, DfE may possibly ease difficulties often accompanied by waste disposal, reducing the disposal costs. On the other hand, environmentally friendly disposal of waste may possibly help production processes become more environmentally friendly by releasing the information on proper treatment of waste, which can improve production processes in a more environmentally friendly way. These are the things which are happening in an actual economy and the points which I would like to emphasize and analyze in this paper.

The structure of this paper is as follows: The next section displays the fundamental settings of the paper. A rough sketch of an economy is given as well as the basic assumptions and structure of the model, to facilitate the understanding of the general thrust of the paper. In the third section, some fundamental mathematical deductions and main propositions are demonstrated. In the final section, concluding remarks are made.

\section{The basic model and assumptions}

\subsection{A rough sketch of the model}

This subsection develops the basic framework of this paper, giving a rough sketch on a whole economy and transactions, based upon which I would like to give a more detailed description by means of an abstract model. The precise formulation of the model is given in the next subsection.

Let us consider an economy where there are three normal production processes and two waste-disposal processes. The first of the normal production processes is one which produces a commodity only for input use (the first commodity), inputting itself and labor. The other two normal production processes (namely the second and the third production processes) are ones which produce a commodity only for consumption use (the second commodity), inputting the first commodity and labor. I assume that the second and the third production processes produce commodities which are regarded as identical at the consumption stage.

There is, however, a difference between those production processes which produce the second commodity. The second production process is environmentally friendly, so that the waste generated through consumption of the commodity produced by the process is easily treated by a waste-disposal process at the post-consumption stage. On the other hand, the third production process is environmentally unfriendly, so that the waste generated through consumption of the commodity produced by this process is treated with difficulty at the post-consumption stage and more inputs are required than the second one for disposal activity. It is assumed that the difference of the two types of waste is not identified by households, and thus, they are discharged in a mixed form, without separation.

A disposal activity consists of two processes: One is an environmentally friendly disposal process (the fourth process), which disposes of waste without any environmental burden (external diseconomy) insofar as it treats the waste of the second commodity which is produced by the environmentally friendly production process (the second process). If it treats the waste of the second commodity which is produced by the environmentally unfriendly production process (the third process), the disposal activity is supposed to create environmental burdens, even if it is an unintentional event. ${ }^{3}$ For

${ }^{3}$ Here, environmental burdens refer to not only external diseconomy in an ordinary sense, but also other damages such as loss of natural capital and so on. 
instance, such a thing happens when an environmentally unfriendly production process uses toxic or hazardous substances but does not release proper information on the product contents.

The other disposal process is an environmentally unfriendly process, which is activated by firms who dispose of waste in an intentionally improper manner. Since this process is intentionally utilized for improper disposal or illegal dumping, it does not matter from which process the waste originates, the second process or the third process. Intentionally improper waste disposal does not care about the information of product contents, just disposing of waste in an improper manner.

As a waste treatment activity, I consider only a waste-disposal process: I do not take up a recycling process explicitly in this paper. Hence, waste generated through consumption is always treated as bads or dis-commodities. This is because I would like to make the model as simple as possible. If one is ready to make a model more complicated, one would not find any difficulty for introducing a recycling process to the present model. In such an economy, waste may possibly be treated as goods.

Apparently, this is a limitation of this paper. A goods/bads relationship should become more important particularly when a dynamic process is taken into account. As Kurz (2008) points out, bads or dis-commodities may be turned to goods by innovation, especially by introduction of a new recycling technology, and it is actually happening in a real economy. However, such a dynamic aspect is out of the scope of the present paper, so that I would like to eschew the problem.

Considering the above, I would like to emphasize that producers of a consumption commodity and disposal firms may work in an interactive manner out of a market boundary on certain conditions as follows. Environmentally friendly producers adopt a production process which gives no or little burden to the environment when that commodity is properly treated by disposal processes at the post-consumption stage. They also release information on the production process, product contents, product composition and so on in a manual, labeling, an Internet service and other measures. These help waste-disposal firms treat the waste in a more efficient and more environmentally friendly way, when they want to do so. Completely the opposite can be applicable to environmentally unfriendly producers, who do not mind adopting a production process which gives an adverse effect to the environment at the post-consumption stage. They do not release any information on the production process, product contents, product composition and so on. Therefore, the waste created by consumption of the commodity produced by these producers is difficult to treat properly and tends to bring about environmental problems at the waste-disposal stage.

Next, let me show how disposal firms affect producers of consumption commodities. Environmentally friendly waste-disposal firms treat waste in a proper way and give no or little burden to the environment. They not only collect the information for completing proper disposal but also release it to producers, asking them to produce a more environmentally friendly commodity, which is disposed of without difficulty at the post-consumption stage. These strengthen the trend of DfE, encouraging producers to produce a more environmentally friendly commodity. It is worth pointing out that this may possibly contribute to the improvement of a production process. Environmentally unfriendly disposal firms ignore these and do not contribute to DfE. 
Working in an interactive or collaborative way as mentioned above, environmentally friendly producers and disposal firms can contribute to constructing a reproducible economy where environmental burdens or environmental costs at the post-consumption stage are reduced as much as possible. This type of interaction or collaboration is seen between automobile manufacturers and disposal firms of end-of-life vehicles (ELVs). Indeed, ACEA (2011) emphasizes this aspect. ${ }^{4}$ Commission European (2014) also shows the importance of such collaboration for enhancing the so-called resource efficiency. Enhancement of resource efficiency is supposed to contribute to prompting producers to adopt not only economically but also environmentally efficient production processes. However, it is worth emphasizing that such interaction or collaboration is possible only when a sort of institutional arrangement is made by the authority.

From the above consideration, let me assume the following: As the activity level of an environmentally friendly production process increases, environmental burdens or environmental costs made by the disposal activity are reduced, since the proportion of unintentionally improper disposal of waste also decreases, possibly due to an increase in proper information of product contents and so on. It may also be considered that coefficients of an environmentally friendly disposal process decrease as the proportion of an environmentally friendly production process to that of an environmentally unfriendly production process increases, since more proper information on product contents and so on is released and supposed to contribute to improvement of an environmentally friendly disposal process.

On the other hand, as the activity level of an environmentally friendly disposal process increases, there is more feedback of information on the proper treatment of waste from an environmentally friendly disposal process to an environmentally friendly production process, so that there is an improvement of production in the process. Surely, more information on how one can properly dismantle and treat waste contributes to the enhancement of DfE. This also implies that coefficients of the production process improve, as the proportion of an environmentally friendly disposal process to an environmentally unfriendly disposal process increases.

\subsection{Formal description of the model}

\subsubsection{Production and disposal processes}

Having given a rough sketch of the model, let me formally express the technology of this economy as follows (see Tables 1, 2):

In Table 1, the first to the third rows describe normal production processes. The first row shows a process which produces a commodity for input use (the first commodity). The second and the third rows show processes which produce a commodity for consumption use (the second commodity), inputting the first commodity and labor. The difference between the processes is that the former process is an environmentally friendly one, giving less environmental burden than the latter process at the post-consumption stage, in the sense that waste created by consumption is smaller in the former process than in the latter process.

$\overline{{ }^{4} \text { A similar type of interaction can be found between manufacturers of electric appliances and recyclers. See Ueno }}$ (2004). 
Table 1 A structure of production of commodities

\begin{tabular}{|c|c|c|c|c|c|c|}
\hline \multirow{2}{*}{$\begin{array}{l}\text { Production (dis- } \\
\text { posal) process }\end{array}$} & \multicolumn{3}{|l|}{ Input } & & \multicolumn{2}{|l|}{ Output } \\
\hline & $\begin{array}{l}\text { A commodity } \\
\text { for input use }\end{array}$ & Waste & Labor & & $\begin{array}{l}\text { A commodity } \\
\text { for input use }\end{array}$ & $\begin{array}{l}\text { Consumption } \\
\text { commodity }\end{array}$ \\
\hline I & $a_{1}$ & 0 & $I_{1}$ & $\rightarrow$ & 1 & 0 \\
\hline$\|$ & $a_{2}(\beta)$ & 0 & $I_{2}(\beta)$ & $\rightarrow$ & 0 & 1 \\
\hline III & $a_{3}$ & 0 & $1 / 3$ & $\rightarrow$ & 0 & 1 \\
\hline IV & $a_{4}(\alpha)$ & 1 & $I_{4}(\alpha)$ & $\rightarrow$ & 0 & 0 \\
\hline V & $a_{5}$ & 1 & $1_{5}$ & $\rightarrow$ & 0 & 0 \\
\hline
\end{tabular}

Table 2 A structure of waste generation

\begin{tabular}{llll}
\hline Consumption commodity (II) & Consumption commodity (III) & & Waste generation \\
\hline 1 & 0 & $\rightarrow$ & $\phi_{2}(\beta)$ \\
0 & 1 & $\rightarrow$ & $\phi_{3}(=1)$ \\
\hline
\end{tabular}

Since the amount of waste generated by consumption of a unit of the commodity produced by the second process is considered to be smaller than that by the third process, thanks to the nature of environmental friendliness as stated above, it is assumed that $\phi_{2}(\beta)<\phi_{3}$ always holds for any $\beta$, where $\beta$ denotes an index to express positive effects from an environmentally friendly disposal process to an environmentally friendly production process, whose formal definition will be given later. If the index increases, DfE is promoted, so that the amount of waste originated in the second process becomes smaller. I may put $\phi_{3}=1$ without loss of generality, and assume that $\phi_{2}(\beta) \in(0,1)$.

The coefficients of inputs of the second process may also depend upon parameter $\beta$ : There is a route of positive effects from an environmentally friendly disposal process to an environmentally friendly production process. For instance, as the share of the environmentally friendly disposal process increases and accordingly $\beta$ increases, there is more positive feedback of the information to the environmentally friendly production process (the second process), say, on how to dismantle and treat ELPs properly. It is also possible that the level of collaboration between two actors increases. Then, the possibility for improvement of the production design becomes greater, since this information contributes to kaizen or improvement. In this way, environmentally friendly waste disposal affects an environmentally friendly production process in an advantageous manner.

Yet, it must be noted that an environmentally friendly production process is inferior to an environmentally unfriendly one insofar as the private cost structure is concerned, since the latter process is never concerned with the environmental burden caused by the waste of the commodity which it produced. Therefore, it is natural to suppose that input coefficients are larger in the second process than in the third process. Hence, I assume that $\left(a_{2}(\beta), l_{2}(\beta)\right)>\left(a_{3}, l_{3}\right)$ holds for any $\beta$ in Table $1 .^{5}$

\footnotetext{
${ }^{5}$ I adopt the following notation for vector inequalities. $x \equiv\left(x_{1}, \ldots, x_{n}\right) \leq y \equiv\left(y_{1}, \ldots, y_{n}\right)$ if $x_{i} \leq y_{i}$ for any $i(=1, \ldots, n)$. $x \ll y$ if $x_{i}<y_{i}$ for any $i(=1, \ldots, n) . x<y$ if $x \leq y$ and $x \neq y$.
} 
The fourth and the fifth processes are disposal processes which treat waste created by consumption. Both processes can treat waste, whichever production process it originated from. Yet, there is a difference between the two processes as follows. The former process is an environmentally friendly disposal process, which does not cause any environmental burden insofar as it treats waste whose origin is in the consumption commodity produced by the second process (an environmentally friendly production process of a consumption commodity). Unintentionally improper disposal is, however, brought about by this process, when the waste comes from the consumption commodity which is produced by the environmentally unfriendly production process (the third production process). This is because proper treatment cannot be made without sufficient information on the production process, product contents and so on, which is released by the second process, but not by the third process.

The coefficients of inputs of the fourth process are assumed to depend upon parameter $\alpha$, which denotes an index to express positive effects from an environmentally friendly production process to an environmentally friendly disposal process, whose formal definition will be given later. As the index increases, the coefficients are supposed to decrease. This is because the disposal activity of proper treatment will improve as circumstances become more favorable for the process.

On the other hand, the fifth process, which is an environmentally unfriendly disposal process, is not affected by the index $\alpha$. Bluntly speaking, it is a disposal process which is utilized when improper disposal of waste is intended. Illegal dumping of waste is a typical example. Thus, it is independent of the index. It must be noted that some inputs are indispensable even for intentionally improper disposal: Labor and material inputs are required for transportation of waste, secret dumping, concealment of bad behavior and so on.

Although I assume that the input coefficients of this process are smaller than those of the former disposal process, that is, $\left(a_{5}, l_{5}\right)<\left(a_{4}(\alpha), l_{4}(\alpha)\right)$ for any $\alpha$, the gaps may be narrow, particularly when policing efforts of the authority are relatively large. Furthermore, the input coefficients $\left(a_{5}, l_{5}\right)$ are supposed to be independent of parameter $\alpha$, since positive effects to proper disposal of waste does not matter for the intentionally improper disposal activity.

\subsubsection{Indices of positive effects between environmentally friendly production and disposal processes}

Here, I would like to define indices of positive effects between environmentally friendly production and disposal processes and explain the environmental burdens given by disposal activities.

As I have already explained in Sect. 2.1, if an activity level of the second process is relatively larger than that of the third process, circumstances are more favorable for the fourth process, i.e., the environmentally friendly disposal process, thanks to positive external effects. Thus, it may be natural to consider that an index of positive effects from the second process to the fourth process depends upon the relative degree of two activity levels, so that $\alpha$ may be expressed as $\alpha \equiv \alpha\left(x_{2}, x_{3}\right)$, where $x_{i}$ denotes an activity level of the $i$-th process. 
Particularly, it should be simpler and more convenient if $\alpha$ would be formulated in a more specific way as follows:

$$
\alpha \equiv \alpha\left(x_{2}, x_{3}\right) \equiv \frac{x_{2}}{x_{2}+x_{3}} \quad\left(x_{2}+x_{3} \neq 0\right),
$$

which is adopted in the following. ${ }^{6}$

Next, let me define $\beta$, which expresses an index of positive effects from the fourth process to the second process. As also already explained, circumstances become more favorable for the second process as an activity level of the fourth process becomes relatively large compared to that of the fifth process. Thus, $\beta$ is supposed to depend upon $\left(x_{4}, x_{5}\right)$, so that $\beta \equiv \beta\left(x_{4}, x_{5}\right)$ holds.

Particularly, it should be simpler and more convenient if $\beta$ would be formulated in a more specific way as follows:

$$
\beta \equiv \beta\left(x_{4}, x_{5}\right) \equiv \frac{x_{4}}{x_{4}+x_{5}} \quad\left(x_{4}+x_{5} \neq 0\right),
$$

which is adopted in the following.

In the following, I often use the notations $\alpha$ and $\beta$ instead of $\alpha\left(x_{2}, x_{3}\right)$ and $\beta\left(x_{4}, x_{5}\right)$ for simplicity, if there is no room for misunderstanding. ${ }^{7}$

Now, let me give a formal definition to the environmental cost caused by environmental burdens made by disposal activities. This consists of two components: One is from unintentionally improper disposal of waste, while the other is from intentionally improper disposal of waste. Denoting activity levels of the two disposal processes as $x_{i}(i=4,5)$, the environmental costs made by the latter may be denoted as $p_{2}^{s} \theta_{5} x_{5}$, where $\theta_{5}$ is a constant parameter, which shows the environmental burdens per activity. Clearly, the environmental costs are expressed in terms of a consumption commodity. ${ }^{8}$

What about the environmental costs made by the former disposal activity? Considering that two types of waste are mixed uniformly, it is reasonable to assume that $\left\{x_{2} \phi_{2}(\beta) /\left(x_{2} \phi_{2}(\beta)+x_{3}\right)\right\} x_{4}$ is the amount of waste which is originated by the environmentally friendly production process and treated by the environmentally friendly disposal process. This does not create any environmental burdens.

On the other hand, $\left[1-\left\{x_{2} \phi_{2}(\beta) /\left(x_{2} \phi_{2}(\beta)+x_{3}\right)\right\}\right] x_{4}$ is the amount of waste which is originated by the environmentally unfriendly production process and treated by the environmentally friendly disposal process. This creates environmental burdens, although it is brought about unintentionally. These costs are expressed as

$$
p_{2}^{s} \theta_{4}\left(1-\frac{x_{2} \phi_{2}(\beta)}{x_{2} \phi_{2}(\beta)+x_{3}}\right) x_{4}
$$

where $\theta_{4}$ is a constant parameter, which shows the environmental burdens per amount of the waste treated. The costs are also valued in terms of a consumption commodity. Let me assume that $\theta_{4}<\theta_{5}$ holds.

\footnotetext{
${ }^{6}$ The functional form can be generalized if some characteristics such as homogenous of degree zero are satisfied. The same consideration is applicable to the form of $\beta$.

7 When I emphasize that $\alpha$ and $\beta$ depend upon $\left(x_{2}, x_{3}\right)$ and $\left(x_{4}, x_{5}\right)$, respectively, I use the notation $\alpha(x)$ and $\beta(x)$.

8 The environmental costs may be valued in terms of any commodity. Yet, it is convenient to express them by a consumption commodity, since it will be adopted as numeraire, namely $p_{2}^{s}=1$ later.
} 
That having been said, I can define the total environmental costs as follows:

$$
p_{2}^{s} \theta_{4}\{1-\psi(x)\} x_{4}+p_{2}^{s} \theta_{5} x_{5}
$$

where $\psi(x) \equiv\left\{x_{2} \phi_{2}(\beta(x)) /\left(x_{2} \phi_{2}(\beta(x))+x_{3}\right)\right\}$. I assume that these environmental costs appear at the end of disposal activities.

\subsubsection{Formal assumptions}

In order to promote smooth development of the following analysis, let me enumerate the assumptions.

Assumption $1 \quad 0<a_{1}<1$ and $g<\frac{1}{a_{1}}-1$.

Assumption $2 a_{2}^{\prime}(\beta)<0, l_{2}^{\prime}(\beta)<0$ and $\phi_{2}^{\prime}(\beta)<0$.

Assumption $3\left(a_{2}(\beta), l_{2}(\beta)\right)>\left(a_{3}, l_{3}\right)$ for $\beta \in[0,1]$.

Assumption $4 \quad a_{4}^{\prime}(\alpha)<0$ and $l_{4}^{\prime}(\alpha)<0$.

Assumption $5\left(a_{4}(0), l_{4}(0)\right) \gg\left(a_{5}, l_{5}\right)$ and $\left(a_{4}(\alpha), l_{4}(\alpha)\right)>\left(a_{5}, l_{5}\right)$ for $\alpha \in[0,1]$.

Let me briefly explain the assumptions: Assumption 1 means a feasibility condition of the whole economy. This is completely the same as the one which is adopted in a conventional linear production model.

The former part of Assumption 2 roughly means that productivity of an environmentally friendly production process increases as the proportion of an activity level of the fourth process to that of the fifth process increases, since positive effects from an environmentally friendly disposal process to an environmentally friendly production process increase. Also, the amount of waste per consumption of an environmentally friendly commodity decreases, since DfE is promoted by an increase in $\beta$ : This is the latter part of Assumption 2.

Assumption 3 implies that productivity of an environmentally friendly production process is smaller than that of an environmentally unfriendly production process. Thus, the former production process is more costly than the latter for producing a unit of consumption commodity.

Assumption 4 roughly means that productivity of an environmentally friendly disposal process increases as the proportion of an activity level of the second process to that of the third process increases, since positive effects from an environmentally friendly production process to an environmentally friendly disposal process increase. This assumption coupled with Assumption 2 means that there are positive interactive effects between environmentally friendly production and disposal processes.

Assumption 5 is a comparison of productivity between environmentally friendly and unfriendly disposal processes. The former part mentions that if the index of positive effects defined by $\alpha$ is zero, productivity of the former disposal process in terms of both material and labor inputs is smaller than that of the latter, or in other words, costs of the former disposal process are larger than those of the latter. This is quite a natural assumption: Intentionally improper disposal is made because it is less costly than proper 
treatment of waste, and this situation is quite likely when there is no environmentally friendly circumstance for proper treatment of waste. The latter part of Assumption 5 says that productivity of the former disposal process, in terms of material and labor input, is not greater than that of the latter process, but either material productivity or labor productivity is smaller in the former process. This is not an artificial assumption, either.

\subsection{Quantity and price formations}

In this subsection, I define quantity systems as well as price systems which must hold in the long run. I present a sustainable equilibrium, a market equilibrium and a socially optimal equilibrium in order.

\subsubsection{Sustainable equilibrium}

First, let me start from the formulation of a quantity system in a long-run competitive equilibrium as follows:

$$
\left\{\begin{array}{l}
(1+g)\left\{a_{1} x_{1}+a_{2}(\beta) x_{2}+a_{3} x_{3}+a_{4}(\alpha) x_{4}+a_{5} x_{5}\right\} \leq x_{1} \\
0<c \leq x_{2}+x_{3} \\
\left\{\phi_{2}(\beta) x_{2}+x_{3}\right\} \leq(1+g)\left(x_{4}+x_{5}\right) \\
l_{1} x_{1}+l_{2}(\beta) x_{2}+l_{3} x_{3}+l_{4}(\alpha) x_{4}+l_{5} x_{5} \leq l
\end{array}\right.
$$

and

$$
\left\{\begin{array}{l}
(1+g)\left\{a_{1} x_{1}+a_{2}(\beta) x_{2}+a_{3} x_{3}+a_{4}(\alpha) x_{4}+a_{5} x_{5}\right\} p_{1}^{s}=x_{1} p_{1}^{s} \\
c p_{2}^{s}=\left(x_{2}+x_{3}\right) p_{2}^{s} \\
\left\{\phi_{2}(\beta) x_{2}+x_{3}\right\} p_{3}^{s}=(1+g)\left(x_{4}+x_{5}\right) p_{3}^{s} \\
\left\{l_{1} x_{1}+l_{2}(\beta) x_{2}+l_{3} x_{3}+l_{4}(\alpha) x_{4}+l_{5} x_{5}\right\} w^{s}=l w^{s},
\end{array}\right.
$$

where $g$ and $x_{i}(i=1, \ldots, 5)$ are a growth rate and an activity level of each process, respectively, and $\left(p_{1}^{s}, p_{2}^{s}, p_{3}^{s}, w^{s}\right)$ is a vector of the production prices and the wage rate, which are formulated later in a dual system.

The first and second equalities of (1) show supply-demand balances for circulating capital and consumption commodities, while the third equality is a supply-demand balance for waste disposal. Incidentally, $0<c \leq x_{2}+x_{3}$ means non-triviality of a solution. It must be noted that two types of waste are discharged in a mixed form by households, since the difference is assumed to be unidentified by them. The last inequality is a labor constraint. Equality system (2) means that the value of supply equals that of demand for each commodity, disposal and labor.

Although inequality system (1) coupled with equality system (2) seems to allow free disposal, it does not actually. This is because $c=x_{2}+x_{3}$ holds by definition, so that this (in)equality may be dropped.

Inequality system (1) and equality system (2) are expressed in matrix form as follows:

$$
A(g, \alpha, \beta) x \leq L \quad \text { and } \quad p^{s} A(g, \alpha, \beta) x=p^{s} L,
$$

where $A(g, \alpha, \beta), L, x$ and $p^{s}$ are defined as 


$$
\begin{aligned}
A(g, \alpha, \beta) & \equiv\left(\begin{array}{ccccc}
(1+g) a_{1}-1 & (1+g) a_{2}(\beta) & (1+g) a_{3} & (1+g) a_{4}(\alpha) & (1+g) a_{5} \\
0 & \phi_{2}(\beta) & 1 & -(1+g) & -(1+g) \\
l_{1} & l_{2}(\beta) & l_{3} & l_{4}(\alpha) & l_{5}
\end{array}\right), \\
L & \equiv\left(\begin{array}{l}
0 \\
0 \\
l
\end{array}\right), x \equiv\left(x_{1}, x_{2}, \ldots, x_{5}\right)^{\mathrm{T}} \text { and } p^{s} \equiv\left(p_{1}^{s}, p_{3}^{s}, w^{s}\right)
\end{aligned}
$$

respectively. ${ }^{9}$

Now, let me refer to a dual system to (1):

$$
\left\{\begin{array}{l}
(1+r) a_{1} p_{1}^{s}+w^{s} l_{1} \geq p_{1}^{s} \\
(1+r) a_{2}(\beta) p_{1}^{s}+\phi_{2}(\beta) p_{3}^{s}+w^{s} l_{2}(\beta) \geq p_{2}^{s} \\
(1+r) a_{3} p_{1}^{s}+p_{3}^{s}+w^{s} l_{3} \geq p_{2}^{s} \\
(1+r)\left(a_{4}(\alpha) p_{1}^{s}-p_{3}^{s}\right)+w^{s} l_{4}(\alpha) \geq-p_{2}^{s} \theta_{4}(1-\psi) \\
(1+r)\left(a_{5} p_{1}^{s}-p_{3}^{s}\right)+w^{s} l_{5} \geq-p_{2}^{s} \theta_{5}
\end{array}\right.
$$

and

$$
\left\{\begin{array}{l}
\left\{(1+r) a_{1} p_{1}^{s}+w^{s} l_{1}\right\} x_{1}=p_{1}^{s} x_{1} \\
\left\{(1+r) a_{2}(\beta) p_{1}^{s}+\phi_{2}(\beta) p_{3}^{s}+w^{s} l_{2}(\beta)\right\} x_{2}=p_{2}^{s} x_{2} \\
\left\{(1+r) a_{3} p_{1}^{s}+p_{3}^{s}+w^{s} l_{3}\right\} x_{3}=p_{2}^{s} x_{3} \\
\left\{(1+r)\left(a_{4}(\alpha) p_{1}^{s}-p_{3}^{s}\right)+w^{s} l_{4}(\alpha)\right\} x_{4}=-p_{2}^{s} \theta_{4}(1-\psi) x_{4} \\
\left\{(1+r)\left(a_{5} p_{1}^{s}-p_{3}^{s}\right)+w^{s} l_{5}\right\} x_{5}=-p_{2}^{s} \theta_{5} x_{5},
\end{array}\right.
$$

where $r$ is a profit rate. In the following, I assume $g=r$ for simplicity.

It must be noted that a base of valuation, namely numeraire, must be chosen in order to determine relative prices. I adopt the consumption commodity as numeraire, setting $p_{2}^{s}=1$. This implies that the environmental costs are valued in terms of this commodity. ${ }^{10}$

The above inequality and equality relationships, namely (4) and (5), are simply written in matrix form as follows:

$$
p^{s} A(r, \alpha, \beta) \geq \Theta(\psi) \quad \text { and } \quad p^{s} A(r, \alpha, \beta) x=\Theta(\psi) x .
$$

It must be noted that $\Theta(\psi)$ is defined as $\Theta(\psi) \equiv\left(0,1,1,-\theta_{4}(1-\psi),-\theta_{5}\right)$.

The first inequality of (4) means a cost-price balance in a circulating capital commodity production process, while the second and the third mean cost-price balances in consumption commodity production processes. The difference between the former and the latter is that the waste-disposal costs are reflected in the left-hand sides of the second and the third inequalities as the terms $\phi_{2}(\beta) p_{3}^{s}$ and $p_{3}^{s}$, although there is no such term in the left-hand side of the first inequality. Here, $p_{3}^{s}$ is interpreted as the price of waste treatment service, and not that of bads or a dis-commodity, which is expressed as $-p_{3}^{s}$.

The fourth and the fifth inequalities of (4) mean cost-price balances in waste-disposal processes. There are two terms which are not found in other inequalities: The first is the

\footnotetext{
${ }^{9}$ In vector notation, $x^{\top}$ denotes transposition of $x$.

10 Obviously, what is adopted as numeraire does not affect the price and quantity systems essentially, since only relative valuation matters.
} 
term $-p_{3}^{s}$ in the left-hand sides of the inequalities. This term reflects a negative cost or positive revenue for disposal activity services at the beginning of disposal activities. The second is the term which shows environmental costs, i.e., $-\theta_{4}(1-\psi)$ and $-\theta_{5}$, in the right-hand side of the inequalities. These costs are expressed in terms the consumption commodity. Thus, the environmental costs caused by the waste-disposal activities are internalized. It might be easier to understand the cost-price balances if terms are transposed, say in the case of the fourth inequality, as follows:

$$
\begin{aligned}
(1+r) a_{4}(\alpha) p_{1}^{s}+\theta_{4}(1-\psi)+w l_{4}(\alpha) & \geq(1+r) p_{3}^{s} \\
(1+r) a_{5} p_{1}^{s}+\theta_{5}+w l_{5} & \geq(1+r) p_{3}^{s} .
\end{aligned}
$$

Notice that environmental costs appear at the end of waste-disposal activities and the revenue for waste-disposal services appears at the beginning of the activities.

The meaning of (5) is clear: Any process which produces deficits is not activated in a long run. Inequality system (4) coupled with equality system (5) shows a constellation of production prices for sustainability, since waste-disposal costs as well as environmental costs are fully taken into account. Under the assumption that $g$ equals $r$, clearly $A(g, \alpha, \beta)$ coincides with $A(r, \alpha, \beta)$, so that the duality between (3) and (6) is clear. Yet, since $\alpha$ and $\beta$ as well as $\psi$ are dependent upon $x$, the duality is different from the one which is expressed by the conventional linear programming problems: The systems expressed by (3) and (6) are nonlinear.

Let me explain the significance of the dual system formulated by (1) with (2) and (4) with (5). First, in this system, the waste treatment costs are reflected in the production of the consumption commodity, from which waste is produced at the post-consumption stage. This is the result of the dual nature between the quantity and price inequalities. Second, environmental costs caused by the waste treatment are expressed in the price inequalities, so that the environmental costs are internalized. Third, the dual system expresses the reproducibility of an economy in a steady state as the conventional linear inequality models. Summing up, I may say that the dual system guarantees the reproducibility of an economy with environmental consideration. Thus, let me call the equilibrium defined by (1) with (2) and (4) with (5) a sustainable equilibrium.

Obviously, a sustainable equilibrium is different from a market equilibrium, which is defined in the next subsection. It is also different from an equilibrium obtained by maximization of the net social benefits, although it may coincide with a sustainable equilibrium on certain conditions, as shown later.

\subsubsection{Market equilibrium}

Let me formulate a market equilibrium in the long run and give a specific meaning to it in the present paper. To do so, I would like to mention the fact that waste is discharged by household consumption activity. Hence, presumably it is legitimate to consider that waste belongs to households, as might be understood in daily life. Then, it is households who have to pay for waste disposal, in whichever form they are charged-a disposal tax or a disposal charge. In the present paper, I assume the latter form: Household waste is transacted between households and disposal firms, so that the former has to pay a disposal charge to the latter. The charge is assumed to be determined in a competitive way. 
Even under this specification, the supply-demand balances in the long run are the same as those in a sustainable equilibrium. The cost-price relationship in a market equilibrium is, however, quite different from those expressed in (4) and (5). It is expressed as follows:

$$
\left\{\begin{array}{l}
(1+r) a_{1} p_{1}^{m}+w^{m} l_{1} \geq p_{1}^{m} \\
(1+r) a_{2}(\beta) p_{1}^{m}+w^{m} l_{2}(\beta) \geq p_{2}^{m} \\
(1+r) a_{3} p_{1}^{m}+w^{m} l_{3} \geq p_{2}^{m} \\
(1+r)\left(a_{4}(\alpha) p_{1}^{m}-p_{3}^{m}\right)+w^{m} l_{4} \geq 0 \\
(1+r)\left(a_{5} p_{1}^{m}-p_{3}^{m}\right)+w^{m} l_{5} \geq 0,
\end{array}\right.
$$

and

$$
\left\{\begin{array}{l}
\left\{(1+r) a_{1} p_{1}^{m}+w^{m} l_{1}\right\} x_{1}=p_{1}^{m} x_{1} \\
\left\{(1+r) a_{2}(\beta) p_{1}^{m}+w^{m} l_{2}(\beta)\right\} x_{2}=p_{2}^{m} x_{2} \\
\left\{(1+r) a_{3} p_{1}^{m}+w^{m} l_{3}\right\} x_{3}=p_{2}^{m} x_{3} \\
\left\{(1+r)\left(a_{4}(\alpha) p_{1}^{m}-p_{3}^{m}\right)+w^{m} l_{4}\right\} x_{4}=0 \\
\left\{(1+r)\left(a_{5} p_{1}^{s}-p_{3}^{m}\right)+w^{m} l_{5}\right\} x_{5}=0 .
\end{array}\right.
$$

In matrix form, the above expressions are written as follows:

$$
p^{m} \tilde{A}(r, \alpha, \beta) \geq \tilde{\Theta} \quad \text { and } \quad p^{m} \tilde{A}(r, \alpha, \beta) x=\tilde{\Theta} x,
$$

where $\tilde{A}(r, \alpha, \beta)$ and $\tilde{\Theta}$ are defined as

$$
\left(\begin{array}{ccccc}
(1+r) a_{1}-1 & (1+r) a_{2}(\beta) & (1+r) a_{3} & (1+r) a_{4}(\alpha) & (1+r) a_{5} \\
0 & 0 & 0 & -(1+r) & -(1+r) \\
l_{1} & l_{2}(\beta) & l_{3} & l_{4}(\alpha) & l_{5}
\end{array}\right) \text { and }(0,1,1,1,0,0)
$$

respectively, and $p^{m}$ is defined as $p^{m} \equiv\left(p_{1}^{m}, p_{3}^{m}, w^{m}\right)$.

Clearly in a market equilibrium, environmental costs are not reflected in the cost-price relationship of disposal processes. Without any constraint on environmental burdens, those environmental costs are never taken into account by waste-disposal firms. Waste treatment costs do not appear in the cost-price relationship of the production processes of a consumption commodity, either. This is because the waste is discharged by households and the treatment charges are on them. Summing up, although environmental costs are not internalized in a market transaction, waste treatment costs are internalized and paid by households instead of producers in a market equilibrium.

To determine relative prices and a wage rate in the above, a commodity or labor must be chosen as numeraire. Since the choice of numeraire does not matter for the relative price determination, I may adopt the consumption commodity as numeraire as before.

Let me make a remark on the payment for waste disposal. Since the waste-disposal charges are supposed to be paid by a household,

$$
w^{m} l=c p_{2}^{m}+\left\{\phi_{2}(\beta) x_{2}+x_{3}\right\} p_{3}^{m}=c p_{2}^{m}+\left\{\alpha \phi_{2}(\beta)+(1-\alpha)\right\} c p_{3}^{m}
$$

must hold in a market equilibrium, since

$$
\phi_{2}(\beta) x_{2}+x_{3}=c\left\{\frac{x_{2}}{x_{2}+x_{3}} \phi_{2}(\beta)+\frac{x_{3}}{x_{2}+x_{3}}\right\}=c\left\{\alpha \phi_{2}(\beta)+(1-\alpha)\right\}
$$


holds. Equation (10) is nothing but a household budget constraint. On the other hand, the constraint in a sustainable equilibrium is

$$
w^{s} l=c p_{2}^{s}-\left\{\theta_{4}(1-\psi) x_{4}+\theta_{5} x_{5}\right\},
$$

since the waste-disposal charges are paid in advance by producers, not by households. Another difference between (10) and (11) is that environmental costs are paid back to households in a sustainable equilibrium, whereas they are not to any actor in a market equilibrium.

It is worth pointing out that the supply-demand balance of a market equilibrium is expressed by means of $\tilde{A}(r, \alpha, \beta)$. Indeed, it is

$$
\tilde{A}(r, \alpha, \beta) x \leq \tilde{L} \quad \text { and } p^{m} \tilde{A}(r, \alpha, \beta) x=p^{m} \tilde{L},
$$

where $\tilde{L}$ is defined as

$$
\tilde{L} \equiv\left(\begin{array}{c}
0 \\
-\left\{\alpha \phi_{2}(\beta)+(1-\alpha)\right\} c \\
l
\end{array}\right) .
$$

As before, it is assumed that $r=g$ and $c=x_{2}+x_{3}$ hold. It is easy to understand that (9) and (12) make a duality.

\subsubsection{Socially optimal equilibrium}

Finally, in this section, I would like to define a socially optimal equilibrium, where the net social benefits are maximized. Notice that I have not referred to any maximization problem so far.

The net social benefits are defined as consumption which is expressed as $x_{2}+x_{3}$ minus the environmental costs which are expressed $\theta_{4}(1-\psi) x_{4}+\theta_{5} x_{5}$. Notice that environmental costs are expressed in terms of a consumption commodity. Hence, the net social benefits expressed as

$$
x_{2}+x_{3}-\left\{\theta_{4}(1-\psi(x)) x_{4}+\theta_{5} x_{5}\right\}=\Theta(\psi(x)) x .
$$

is also expressed in terms of a consumption commodity.

Then, a socially optimal solution can be obtained by solving

$$
\max \Theta(\psi(x)) x \text { s.t. } A(g, \alpha(x), \beta(x)) x \leq L .
$$

\section{Main results}

In this section, let me show the main results: Existence of equilibria and their characteristics. I would also like to compare three types of equilibria, namely a sustainable equilibrium, a market equilibrium and a socially optimal equilibrium.

\subsection{Existence of equilibria}

If there were no equilibrium solution, it should be nonsense to talk about the characteristics of solutions and relationships among equilibria. Thus, first, I show the fundamental existence propositions as follows: 
Proposition 1 There is a solution to (3) and (6), and so a sustainable equilibrium exists.

Proof See "Appendix 1."

Proposition 2 There is a solution to (9) and (12), and so a market equilibrium exists.

Proof See "Appendix 1."

As for the socially optimal equilibrium, it is obvious that there exists an equilibrium solution. This is because constraint (13) is feasible and the maxim and is a continuous function of $x$.

\subsection{Characteristics of equilibria}

Next, let me consider characteristics of equilibria. First, consider a sustainable equilibrium. Since the second process is an environmentally friendly production process and the fourth process is an environmentally friendly disposal process, it might be thought that they should be activated instead of the third process and the fifth process, which are environmentally unfriendly and jointly bring about environmental burdens.

This is not true in a general case. If the coefficient of environmental costs is very small, it may be reasonable to activate the fifth process, since it is less costly than the fourth process. Moreover, the third process may be activated when $\beta$ is not so large, due to Assumption 3.

Yet, it is expected that the third and the fifth processes are not activated if environmental costs caused by improper treatment of waste are so large, whether it is intentional or not. Actually, this expectation is correct as follows:

Proposition 3 In a sustainable equilibrium, $x_{3}^{*}$ and $x_{5}^{*}$ go to zero as $\theta_{4}$ and $\theta_{5}$ go to infinity.

Proof See "Appendix 2."

From the argument of continuity, it is known that $x_{3}^{*}$ and $x_{5}^{*}$ are sufficiently small if $\theta_{i}(i=4,5)$ is sufficiently large. Thus, if environmental costs caused by improper treatment of waste are very large, the environmentally unfriendly production process and disposal process are not activated in a sustainable equilibrium.

It is natural to expect to obtain a different result from the above in a market equilibrium, where environmental costs are not reflected in the cost-price relationship. Actually, I can show the following result:

Proposition 4 In a market equilibrium, $x_{2}^{m *}=x_{4}^{m *}=0$.

Proof See "Appendix 2."

The message of this proposition is clear: Without any constraint upon environmental burdens, both environmentally friendly production and disposal processes are knocked out, and only environmentally unfriendly production and disposal processes remain 
in an economy. Therefore, there are no positive interactive effects between production and disposal processes. This is a natural result which comes from the cost-minimizing principle and should be compared to that of Proposition 3. As I show later, extended producer responsibility realizes the sustainable equilibrium, so that it internalizes the external diseconomy partly. Furthermore, under certain conditions, it internalizes the external diseconomy completely, realizing the social optimum.

It must be noted that Proposition 3 requires the condition that $\theta_{i}(i=4,5)$ goes to infinity or is sufficiently large. Let me consider the opposite case, i.e., the case in which $\theta_{i}(i=4,5)$ is very small. The extreme case is when $\theta_{i}(i=4,5)$ is zero. I can show the following:

Proposition 5 Suppose $\theta_{i}(i=4,5)$ is sufficiently small, so that it is close to zero. Then, $x_{4}^{*}=0$ and $x_{5}^{*}>0$ in a sustainable equilibrium. Furthermore, (1) if $l_{1}$ is sufficiently small and the rate of profit is close to its maximum $\left(=1 / a_{1}-1\right)$ or (2) if both $\left|a_{2}(0)-a_{3}\right|$ and $\left|l_{2}(0)-l_{3}\right|$ are sufficiently small, then, $x_{2}^{*}>0$ and $x_{3}^{*}=0$, and thus, $\alpha^{*}=1$ in a sustainable equilibrium.

Proof See "Appendix 2."

Hence, an environmentally friendly production process may remain in an economy and an environmentally unfriendly production process may be knocked out even if environmental costs are sufficiently small in a sustainable equilibrium. Yet, in the same circumstances, an environmentally friendly disposal process disappears whereas an environmentally unfriendly disposal process is activated. The effect of the magnitude of environmental costs is asymmetrical between production and disposal processes.

\subsection{Comparison of equilibria}

In this subsection, I would like to compare equilibria in terms of net social benefits. First, let me compare the net social benefits in a sustainable equilibrium and those in a market equilibrium. Although the following proposition may seem obvious, it is not. In a sustainable equilibrium, external costs are internalized, but not in the optimal way. Thus, it must be proved rigorously anyhow:

Proposition 6 The net welfare in a sustainable equilibrium is not smaller than that in a market equilibrium.

Proof From Proposition 4, we know $\alpha^{m *}=\beta^{m *}=0$. Thus, we have

$$
\left(a_{2}\left(\beta^{*}\right), l_{2}\left(\beta^{*}\right)\right) \leq\left(a_{2}\left(\beta^{m *}\right), l_{2}\left(\beta^{m *}\right)\right)
$$

from Assumption 2. Furthermore, from Assumption 4, we know that the following holds:

$$
\left(a_{4}\left(\alpha^{*}\right), l_{4}\left(\alpha^{*}\right)\right) \leq\left(a_{4}\left(\alpha^{m *}\right), l_{4}\left(\alpha^{m *}\right)\right) .
$$

Therefore, the following holds:

$$
A\left(g, \alpha^{*}, \beta^{*}\right) x^{m *} \leq A\left(g, \alpha^{m *}, \beta^{m *}\right) x^{m *} \leq L,
$$


which means

$$
\Theta\left(\psi^{*}\right) x^{*} \geq \Theta\left(\psi^{*}\right) x^{m *} \geq \Theta\left(\psi^{m *}\right) x^{m *},
$$

since $x^{m *}$ is feasible for the problem $\max \Theta\left(\psi\left(x^{*}\right)\right) x$ s.t. $A\left(g, \alpha^{*}, \beta^{*}\right) \leq L$, thanks to (14), and since

$$
\Theta\left(\psi^{m *}\right)=\Theta(0) \equiv(0,1,1,-\theta,-\theta) \leq\left(0,1,1,-\theta\left(1-\psi^{*}\right),-\theta\right) \equiv \Theta\left(\psi\left(x^{*}\right)\right)
$$

holds.

It is interesting to see that I have not utilized Assumptions 2 and 4 which express positive interactive effects between the second and fourth processes to prove the above proposition. Even without these effects, the net social benefits in a sustainable equilibrium are not smaller than those in a market equilibrium, due to the nature of a sustainable equilibrium.

It is clear that the net social benefits in a sustainable equilibrium are not larger than those in a socially optimal equilibrium by definition, so that we have

$$
\Theta\left(\psi\left(x^{m *}\right)\right) \leq \Theta\left(\psi\left(x^{*}\right)\right) \leq \Theta\left(\psi\left(x^{* *}\right)\right),
$$

where $\psi\left(x^{* *}\right)$ corresponds to the socially optimal solution.

Then, a natural question would be this: In what condition are the net social benefits in a sustainable equilibrium larger than those in a market equilibrium and hopefully equal to those in a socially optimal equilibrium? I can answer this question as follows:

Proposition 7 If externality represented by coefficient $\theta_{5}$ is sufficiently large, then the net social benefits in a sustainable equilibrium are larger than those in a market equilibrium.

Proof By the nature of duality, the net social benefits in a sustainable equilibrium cannot be negative, so that $\Theta\left(\psi\left(x^{*}\right)\right) x^{*} \geq 0$ holds. ${ }^{11}$ On the other hand, due to Proposition 4, we have

$$
\Theta\left(\psi\left(x^{m *}\right)\right) x^{m *}=x_{3}^{m *}-\theta_{5} x_{5}^{m *} .
$$

Notice that $x^{m *}$ does not depend upon $\theta_{5}$, and $x_{5}^{m *}$ is calculated as

$$
x_{5}^{m *}=\frac{\left\{1-(1+g) a_{1}\right\} l}{(1+g)\left\{(1+g) a_{3}+a_{5}\right\} l_{1}+\left\{1-(1+g) a_{1}\right\}\left\{(1+g) l_{3}+l_{5}\right\}}>0 .
$$

Thus, $\Theta\left(\psi\left(x^{m *}\right)\right)$ becomes negative as $\theta_{5}$ becomes sufficiently large. Hence, $\Theta\left(\psi\left(x^{*}\right)\right) x^{*}>\Theta\left(\psi\left(x^{m *}\right)\right)$ for sufficiently large $\theta_{5}$.

Next, let me consider the case in which $\theta_{5}$ is sufficiently small, or the case in which environmental costs caused by waste disposal are negligible. I can show the following proposition, which corresponds to Proposition 5:

${ }_{11}$ Actually, this is positive, as shown in the proof of Proposition 1. 
Proposition 8 Suppose both $l_{1}$ and $\theta_{5}$ are sufficiently small, and the rate of profit is close to its maximum. Moreover, if $1-\phi_{2}(0)>(1+g) \max \left(\frac{a_{2}(0)-a_{3}}{a_{5}}, \frac{l_{2}(0)-l_{3}}{l_{5}}\right)$ holds, then $\Theta\left(\psi\left(x^{*}\right)\right) x^{*}>\Theta\left(\psi\left(x^{m *}\right)\right) x^{m *}$.

Proof See "Appendix 3."

What does the above proposition mean? It must be noted that

$$
1-\phi_{2}(0) \equiv \phi_{3}-\phi_{2}(0)>(1+g) \max \left(\frac{a_{2}(0)-a_{3}}{a_{5}}, \frac{l_{2}(0)-l_{3}}{l_{5}}\right)
$$

means that more inputs are required for waste treatment if the third process, instead of the second process, is activated for production of a consumption commodity. ${ }^{12}$ Since $l_{1}$ is assumed to be very small, the wage rate is not so small even if the rate of profit is close to its maximum. Considering these together, activation of the third process instead of the second one is too costly when the waste treatment costs are taken into account. Therefore, the net social benefits in a sustainable equilibrium are larger than those in a market equilibrium.

Finally, I would like to demonstrate the condition in which a sustainable equilibrium coincides with a socially optimal equilibrium for given environmental burdens $\theta_{i}(i=4,5)$. Namely, we have

Proposition 9 A sustainable equilibrium coincides with a socially optimal equilibrium, (i) if both $\theta_{4}$ and $\theta_{5}$ are sufficiently large or (ii) if $\left|a_{j}-a_{i}(0)\right|<\varepsilon$ and $\left|l_{j}-l_{i}(0)\right|<\varepsilon$ holds for sufficiently small $\varepsilon$, where $(i, j)=(2,3)$ and $(4,5)$, respectively.

Proof See "Appendix 4."

\section{Discussion}

Here, I would like to consider the significance of a sustainable equilibrium in the context of producer's responsibility for waste generation. Notice that waste treatment charges are included in production costs in this equilibrium. Of course, the waste treatment charges are reflected in the price of the consumption commodity. Thus, although households do not have to pay for waste treatment directly, they pay indirectly, since those costs are reflected in the production price. In other words, households are supposed pay for waste treatment of an ELP (end-of-life product) in advance when they buy the relevant commodity or product.

This way of payment for waste treatment is interpreted as an application of extended producer responsibility (EPR), which was proposed by OECD. Precisely speaking, it is financial EPR, since producers have to take waste treatment costs into account in their production, accepting the financial burden for waste treatment, although they are not responsible for waste treatment activities.

\footnotetext{
${ }^{12}$ This inequality implies that $\left\{1-\phi_{2}(0)\right\} a_{5} \equiv\left\{\phi_{3}-\phi_{2}(0)\right\} a_{5}>(1+g)\left\{a_{2}(0)-a_{3}\right\}$ and $\left\{1-\phi_{2}(0)\right\} / 5 \equiv\left\{\phi_{3}-\phi_{2}(0)\right\} / 5>(1+g)\left\{/ 2(0)-I_{3}\right\}$ hold.
} 
Considering the above, it is clear that a sustainable equilibrium has a special meaning as an application of EPR in the field of an environmental policy. Since the concept of a sustainable equilibrium is very close to that of the natural price or production price system, it may be said that the classical concept of prices has very important meaning in an environmental policy.

The importance of the classical concept of a price system is quite naturally understood, since the concept is based upon reproducibility of an economic system. If the concept is extended so that environmental restrictions are taken into account, it may be interpreted as sustainability of an economic system. This tells us that the classical concept of a price system is still applicable to a quite modern environmental problem.

\section{Concluding remarks}

In this paper, interactive aspects between environmentally friendly producers and disposal firms are emphasized and analyzed. Although this interaction does not appear in a market equilibrium in which waste treatment charges are on households while environmental costs are not internalized in the production, it does appear in a specific type of equilibrium which is defined as a sustainable equilibrium in this paper.

A sustainable equilibrium is defined based upon the idea of a classical type of production price or natural price system. A difference between a sustainable equilibrium and a classical equilibrium is that environmental factors are explicitly taken into account in the former equilibrium.

I have also demonstrated that the net social benefits of a sustainable equilibrium are between those of a market equilibrium and a socially optimal equilibrium. In special cases, the net social benefits in a sustainable equilibrium are larger than those in a market equilibrium. Moreover, a sustainable equilibrium coincides with a socially optimal equilibrium, when external diseconomy caused by waste treatment services is sufficiently large, or when the relative cost structures in physical terms are not different between environmentally friendly and unfriendly production processes and between environmentally friendly and unfriendly waste treatment processes.

To demonstrate these results, I have utilized Assumptions 2 and 4, which express positive interactive effects between environmentally friendly producers and disposal firms. These effects are created by their collaboration or cooperation whether it may be systematically organized or not. I may say that the present model captures the new trend of collaboration or cooperation between producers and disposal firms which has recently appeared, say in ELV recycling, and shows how it is strengthened in a sustainable equilibrium.

The concept of a sustainable equilibrium, which is akin to the classical concept of natural prices or production prices, can be interpreted as an application of EPR. In this sense, the classical concept of natural prices or production prices has wide applicability to environmental problems. I believe that it is my contribution to the modern classical economics that I demonstrate this result. 


\section{Appendix 1}

Proof of Proposition 1 First, I prove that (3) and (6) have a non-trivial solution for $L_{q} \equiv(0, q, l)^{\mathrm{T}}$ instead of $L$, where $q$ is a positive number. Next, I show that there is a non-trivial solution for $q=0$.

Part 1. Step 1. Let me define a set $X$ as

$$
X \equiv\left\{x \mid 0 \leq x \text { and } l_{1} x_{1}+l_{2}(1) x_{2}+l_{3} x_{3}+l_{4}(1) x_{4}+l_{5} x_{5} \leq l\right\},
$$

which is compact and convex. Let me also define functions $\alpha_{\epsilon}(x), \beta_{\epsilon}(x)$ and $\psi_{\epsilon}(x)$ as

$$
\alpha_{\epsilon}(x) \equiv \frac{x_{2}}{x_{2}+x_{3}+\epsilon}, \quad \beta_{\epsilon}(x) \equiv \frac{x_{4}}{x_{4}+x_{5}+\epsilon} \text { and } \psi_{\epsilon}(x) \equiv \frac{\phi_{2}\left(\beta_{\epsilon}(x)\right) x_{2}}{\phi_{2}\left(\beta_{\epsilon}(x)\right) x_{2}+x_{3}+\epsilon},
$$

where $\epsilon$ is a sufficiently small positive constant. Thus, $\alpha_{\epsilon}(x), \beta_{\epsilon}(x)$ and $\psi_{\epsilon}(x)$ are well defined for any $x \in X$.

Choose $x^{\prime} \in X$, and assign it to $\alpha_{\epsilon}(x), \beta_{\epsilon}(x)$ and $\psi_{\epsilon}(x)$, which are denoted as $\alpha_{\epsilon}^{\prime} \equiv \alpha_{\epsilon}\left(x^{\prime}\right), \beta_{\epsilon}^{\prime} \equiv \beta_{\epsilon}\left(x^{\prime}\right)$ and $\psi_{\epsilon}^{\prime} \equiv \phi_{2}\left(\beta_{\epsilon}^{\prime}\right) x_{2}^{\prime} /\left\{\phi_{2}\left(\beta_{\epsilon}^{\prime}\right) x_{2}^{\prime}+x_{3}^{\prime}+\epsilon\right\}$, respectively. Assign $\left(\alpha_{\epsilon}^{\prime}, \beta_{\epsilon}^{\prime}\right)$ and $\psi_{\epsilon}^{\prime}$ to $A\left(g, \alpha_{\epsilon}, \beta_{\epsilon}\right)\left(\equiv A\left(r, \alpha_{\epsilon}, \beta_{\epsilon}\right)\right)$ and $\Theta\left(\psi_{\epsilon}\right)$.

Then, we can consider the following linear programming problems:

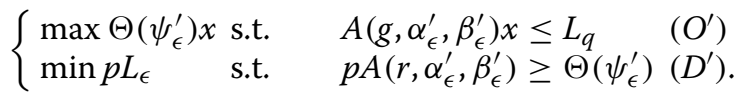

Apparently, $\left(D^{\prime}\right)$ is dual to $\left(O^{\prime}\right)$.

We can find $(\varepsilon, 0,0,0,0)^{\mathrm{T}}$ such that $\varepsilon$ is positive and sufficiently small, and

$$
A\left(g, \alpha_{\epsilon}^{\prime}, \beta_{\epsilon}^{\prime}\right)(\varepsilon, 0,0,0,0)^{\mathrm{T}}=\left(\begin{array}{c}
\left\{(1+g) a_{1}-1\right\} \varepsilon \\
0 \\
l_{1} \varepsilon
\end{array}\right) \leq L_{q}=\left(\begin{array}{l}
0 \\
q \\
l
\end{array}\right),
$$

is satisfied. This means that $\left(O^{\prime}\right)$ is feasible.

Next, choose sufficiently large positive $\delta$,

$(0,0, \delta) A\left(r, \alpha_{\epsilon}^{\prime}, \beta_{\epsilon}^{\prime}\right)=\left(\delta l_{1}, \delta l_{2}\left(\beta_{\epsilon}^{\prime}\right), \delta l_{3}, \delta l_{4}\left(\alpha_{\epsilon}^{\prime}\right), \delta l_{5}\right) \geq\left(0,1,1,-\theta_{4}\left(1-\psi_{\epsilon}^{\prime}\right),-\theta_{5}\right)=\Theta\left(\psi_{\epsilon}^{\prime}\right)$,

which means $\left(D^{\prime}\right)$ is feasible.

The above argument shows that both $\left(O^{\prime}\right)$ and $\left(D^{\prime}\right)$ are feasible, so that both problems have solutions for pre-assigned $x^{\prime}$ (Gale 1960, p. 78).

Step 2. Denoting the solution set of $x$ as $X_{\epsilon}^{*}\left(x^{\prime}\right)$, we know that there is a correspondence $\Psi$ such that

$$
\Psi: x^{\prime} \in X \mapsto X_{\epsilon}^{*}\left(x^{\prime}\right) \in X .
$$

Since $\left(O^{\prime}\right)$ is linear for given $x^{\prime}$, any convex combination of solutions is a solution to $\left(O^{\prime}\right)$, so that $X_{\epsilon}^{*}\left(x^{\prime}\right)$ is convex. Moreover, it is bounded, since $X_{\epsilon}^{*}\left(x^{\prime}\right) \subset X$. Clearly, it is closed, so that $X_{\epsilon}^{*}\left(x^{\prime}\right)$ is compact.

Now, I show that $\Psi$ is upper-hemi-continuous. Consider a sequence $\left\{x_{v}^{\prime}\right\}$ such that $x_{v}^{\prime} \in X$ and $x_{v}^{\prime} \rightarrow x_{0}^{\prime}$. Then, we can choose a sequence $x_{v}^{*}$ such that $x_{v}^{*} \in X_{\epsilon}^{*}\left(x_{v}^{\prime}\right)$ and $x_{v}^{*} \rightarrow x_{0}^{*}$ as $v \rightarrow \infty$. By construction, $x_{0}^{*}$ is a solution to $\left(O^{\prime}\right)$ for $x_{0}^{\prime}$, and so $x_{0}^{*} \in X_{\epsilon}^{*}\left(x_{0}^{\prime}\right)$. 
Otherwise, in the neighborhood of $x_{0}^{*}, \Theta\left(\psi_{\epsilon}^{\prime}\right) x_{v}^{*}<p_{v}^{*} L_{\epsilon}$ should hold, where $p_{v}^{*}$ corresponds to $x_{v}^{*}$. But this is a contradiction. Hence, $\Psi$ is upper-hemi-continuous.

Thanks to Kakutani's fixed point theorem, we know that $\exists x_{\epsilon}^{*} \in X_{\epsilon}^{*}\left(x_{\epsilon}^{*}\right)$ such that

$A\left(g, \alpha_{\epsilon}^{*}, \beta_{\epsilon}^{*}\right) x_{\epsilon}^{*} \leq L_{q}$ and $\Theta\left(\psi_{\epsilon}^{*}\right) x^{*} \geq \Theta\left(\psi_{\epsilon}^{*}\right) x$ for any $x \geq 0$ such that $A\left(g, \alpha_{\epsilon}^{*}, \beta_{\epsilon}^{*}\right) x \leq L_{q}$,

where $\alpha_{\epsilon}^{*} \equiv \alpha\left(x_{\epsilon}^{*}\right), \beta_{\epsilon}^{*} \equiv \beta\left(x_{\epsilon}^{*}\right)$, and $\psi_{\epsilon}^{*} \equiv \psi\left(x_{\epsilon}^{*}\right)$.

Clearly, the dual system $\left(D^{\prime}\right)$ has a solution, corresponding to $x_{\epsilon}^{*}: \exists p_{\epsilon}^{*}>0$ such that

$p_{\epsilon}^{*} A\left(r, \alpha_{\epsilon}^{*}, \beta_{\epsilon}^{*}\right) \geq \Theta\left(\psi_{\epsilon}^{*}\right)$ and $p_{\epsilon}^{*} L_{q} \leq p L_{q}$ for any $p \geq 0$ such that $p A\left(r, \alpha_{\epsilon}^{*}, \beta_{\epsilon}^{*}\right) \leq \Theta\left(\psi_{\epsilon}^{*}\right)$.

Now, let us get $\epsilon \rightarrow 0$. Since $\left\{x_{\epsilon}^{*}\right\}$ is in a compact set $X$, the sequence has an accumulation point $x^{* *} \in X$, which must be a solution to (3). A price vector $p^{* *}$ which corresponds to $x^{* *}$ must also be a solution to (6).

Step 3. Let me show non-triviality of an equilibrium solution. For any given $(\alpha, \beta, \psi) \in[0,1] \times[0,1] \times[0,1]$, consider the following problem:

$\max \Theta(\psi) x \quad$ st. $A(g, \alpha, \beta) x \leq L_{q}$.

Choose $x$ which satisfies $x_{3}=x_{4}=x_{5}=0$ and $0<x_{2} \leq\left\{1-(1+g) a_{1} x_{1}\right\} /(1+g) a_{2}(\beta)$ as well as $x_{2} \leq q / \phi_{2}(\beta)$ and $l_{1} x_{1}+l_{2}(\beta) x_{2}+l_{3} x_{3}+l_{4}(\alpha) x_{4}+l_{5} x_{5} \leq l$. Thus, the above problem is feasible, and $\Theta(\psi) x>0$. Consequently, the maximum value of the above problem is positive and a continuous function of $(\alpha, \beta, \psi)$. Clearly, it has a positive lower bound. This implies that $\Theta\left(\psi_{\epsilon}\right)$ never converges to zero as $\epsilon \rightarrow 0$. Hence, $x_{2}^{*}+x_{3}^{*}>0$.

Part 2. Now, let me show that (3) and (6) have a solution when $q$ equals zero. From the above argument, we know that $x_{2}^{*}+x_{3}^{*}>0$ holds for any positive $q$. Suppose that $q$ converges to zero, and that $x^{*}$ has an accumulation point such that $x_{2}+x_{3}=0$. Then, $x_{2}^{*}+x_{3}^{*}$ must be sufficiently small for sufficiently small $q$.

Case 1. $\phi_{2}(\beta) x_{2}^{*}+x_{3}^{*}<(1+g)\left(x_{4}^{*}+x_{5}^{*}\right)+q$.

Then, $p_{3}=0$ holds. Thus,

$$
\begin{aligned}
(1+r) a_{2}(\beta) p_{1}^{s}+w^{s} l_{2}(\beta) \geq p_{2}^{s} \quad(=1) \\
(1+r) a_{3} p_{1}^{s}+w^{s} l_{3} \geq p_{2}^{s} \quad(=1)
\end{aligned}
$$

hold. Therefore, at least one of $p_{1}^{s}$ and $w^{s}$ is positive.

1. Suppose $p_{1}^{s}>0$ and $w^{s}>0$. From Assumption 3,

$(1+r) a_{2}(\beta) p_{1}^{s}+w^{s} l_{2}(\beta)>(1+r) a_{3} p_{1}^{s}+w^{s} l_{3} p_{2}^{s}(=1)$

holds, so that $x_{2}^{*}=0$, and so $\psi\left(x^{*}\right)=0$ hold. Since $\Theta\left(x^{*}\right) \equiv x_{3}^{*}-\theta_{4} x_{4}^{*}-\theta_{5} x_{5}^{*}$ must be positive, we know that $x_{4}^{*}$ and $x_{5}^{*}$ must be sufficiently small. Notice that

$$
l_{1} x_{1}^{*}+l_{2}(\beta) x_{2}^{*}+l_{3} x_{3}^{*}+l_{4}(\alpha) x_{4}^{*}+l_{5} x_{5}^{*}=l
$$

holds, since $w^{s}>0$ holds by hypothesis. Thus, $x_{1}^{*}$ is relatively large compared to $x_{i}(i=2, \ldots, 5)$, and $x_{1}^{*} \cong l / l_{1}$. But this means

$$
(1+g)\left\{a_{2}(\beta) x_{2}^{*}+a_{3} x_{3}^{*}+a_{4}(\alpha) x_{4}^{*}+a_{5} x_{5}^{*}\right\}<\left\{1-(1+g) a_{1}\right\} x_{1}^{*},
$$

from which we have $p_{1}^{s}=0$. A contradiction.

2. Suppose $w^{s}>0$ and $p_{1}^{s}=0$. Since

$(1+r) a_{1} p_{1}^{s}+w^{s} l_{1}>p_{1}^{s}=0$ 
holds, so that we have $x_{1}^{*}=0$. A contradiction.

3. Suppose $w^{s}=0$ and $p_{1}^{*}>0$. We have

$0=w^{s} l_{1} \geq\left\{1-(1+r) a_{1}\right\} p_{1}^{s}>0$.

A contradiction.

Case 2. $\phi(\beta) x_{2}^{*}+x_{3}^{*}=(1+g)\left(x_{4}^{*}+x_{5}^{*}\right)+q$.

Since $x_{2}^{*}+x_{3}^{*}$ is sufficiently small, $x_{4}^{*}+x_{5}^{*}$ is also sufficiently small.

1. Suppose

$l_{1} x_{1}^{*}+l_{2}(\beta) x_{2}^{*}+l_{3} x_{3}^{*}+l_{4}(\alpha) x_{4}^{*}+l_{5} x_{5}^{*}<l$

holds. Then, $w^{s}=0$, and so $p_{1}^{s}=0$ hold. From the second and third inequalities of (4), we have

$p_{3}^{s} \geq \phi_{2}(\beta) p_{3}^{s}>p_{2}^{s}=1$,

which implies that $x_{3}^{*}=0$ and $x_{2}^{*}>0$.From the fourth and fifth inequalities of (4),

$-(1+r) p_{3}^{s} \geq-p_{2}^{s} \theta_{4}\left(1-\psi\left(x^{*}\right)\right)>-p_{2}^{s} \theta_{5} x_{5}^{*}$

holds. Hence, we have $x_{4}^{*}>0$ and $x_{5}^{*}=0$, so that we know $\beta\left(x^{*}\right)=1$. Therefore, we have $\psi\left(x^{*}\right)=1$. On the other hand, from the above inequality and $\psi\left(x^{*}\right)=1$, we have $-(1+r) p_{3}^{s} \geq 0$, which implies $p_{3}^{s} \leq 0$. A contradiction.

2. Suppose

$l_{1} x_{1}^{*}+l_{2}(\beta) x_{2}^{*}+l_{3} x_{3}^{*}+l_{4}(\alpha) x_{4}^{*}+l_{5} x_{5}^{*}=l$

holds. Then, $x_{1}^{*} \cong l / l_{1}$, and $x_{i}(i=2, \ldots, 5)$ is sufficiently small. Thus,

$(1+g)\left\{a_{2}(\beta) x_{2}^{*}+a_{3} x_{3}^{*}+a_{4}(\alpha) x_{4}^{*}+a_{5} x_{5}^{*}\right\}<\left\{1-(1+g) a_{1}\right\} x_{1}^{*}$,

holds, so that $p_{1}^{s}=0$ holds. Therefore, $w^{s}=0$ holds: Otherwise,

$(1+r) a_{1} p_{1}^{s}+w^{s} l_{1}>p_{1}^{s}=0$,

and so $x_{1}^{*}=0$ hold. A contradiction. Hence, we have $w^{s}=0$. Now, we can apply the same argument as in Case 2 (ii) above to the present case.

Proof of Proposition 2 Notice that (9) and (12) are equivalent to

$$
\begin{cases}p^{m} A(r, \alpha, \beta) \geq \hat{\Theta}\left(\beta, p_{3}^{m}\right) & \text { and } p^{m} A(r, \alpha, \beta) x=\hat{\Theta}\left(\beta, p_{3}^{m}\right) x \\ A(g, \alpha, \beta) x \leq L & \text { and } p^{m} A(g, \alpha, \beta) x=p^{m} L\end{cases}
$$

where $\hat{\Theta}\left(\beta, p_{3}^{m}\right) \equiv\left(0,1+\phi_{2}(\beta) p_{3}^{m}, 1+p_{3}^{m}, 0,0\right)$.

First, let me show that $w^{m}$ is bounded. Suppose that $w^{m}$ is sufficiently large. Then, from the first inequality of (7) and the first equality of (8), we have $x_{1}^{m}=0$, and so $x_{2}^{m}=\cdots=x_{5}^{m}=0$ from the first inequality of (1). This implies that $\sum_{1}^{5} l_{i} x_{i}^{m}=0<l$ and thus, $w^{m}=0$ holds. A contradiction.

Let me denote an upper bound of $w^{m}$ as $\bar{w}^{m}$. Then, the following must hold:

$$
p_{1}^{m} \leq \frac{w^{m} l_{1}}{1-(1+r) a_{1}} \leq \frac{\bar{w}^{m} l_{1}}{1-(1+r) a_{1}},
$$


from which we know

$$
\exists \bar{p}_{1}^{m}>0 \quad \text { s.t. } \quad \bar{p}_{1}^{m} \geq p_{1}^{m} \quad \text { for } \quad r \in[0, R),
$$

where $R \equiv 1 / a_{1}-1$.

From the fifth inequality of the price inequality system in (15), we have

$$
p_{3}^{m} \leq \frac{(1+r) a_{5} p_{1}^{m}+w^{m} l_{5}}{1+r},
$$

which implies

$$
\exists \bar{p}_{3}^{m}>0 \quad \text { s.t. } \quad \bar{p}_{3}^{m}>p_{3}^{m} .
$$

Then, define $P_{3}^{m}$ as

$$
P_{3}^{m} \equiv\left\{p_{3}^{m} \mid 0 \leq p_{3}^{m} \leq \bar{p}_{3}^{m}\right\}
$$

and choose $\left(x^{\prime T}, p_{3}^{\prime m}\right) \in X \times P_{3}^{m}$, assigning it to $A(r, \alpha, \beta)$ and $\hat{\Theta}$. Solve

$$
\left\{\begin{array}{l}
p^{m} A\left(r, \alpha\left(x^{\prime}\right), \beta\left(x^{\prime}\right)\right) \geq \hat{\Theta}\left(\beta\left(x^{\prime}\right), p_{3}^{\prime m}\right) \\
p^{m} A\left(r, \alpha\left(x^{\prime}\right), \beta\left(x^{\prime}\right)\right) x=\hat{\Theta}\left(\beta\left(x^{\prime}\right), p_{3}^{\prime m}\right) x \\
\text { and } \\
A\left(g, \alpha\left(x^{\prime}\right), \beta\left(x^{\prime}\right)\right) x \leq L \\
p^{m} A\left(g, \alpha\left(x^{\prime}\right), \beta\left(x^{\prime}\right)\right) x=p^{m} L,
\end{array}\right.
$$

which is equivalent to

$$
\begin{cases}\max \hat{\Theta}\left(\beta\left(x^{\prime}\right), p_{3}^{\prime m}\right) x & \text { s.t. } A\left(g, \alpha\left(x^{\prime}\right), \beta\left(x^{\prime}\right)\right) x \leq L \\ \min p^{m} L & \text { s.t. } p^{m} A\left(r, \alpha\left(x^{\prime}\right), \beta\left(x^{\prime}\right)\right) \geq \hat{\Theta}\left(\beta\left(x^{\prime}\right), p_{3}^{\prime m}\right) .\end{cases}
$$

Both problems of (16) are feasible, so that they are solvable: They have solutions $x$ and $p^{m}$, respectively for pre-assigned $\left(x^{\prime T}, p_{3}^{\prime m}\right) \in X \times P_{3}^{m}$. Hence, we have correspondence $\Psi$ such that

$$
\Psi:\left(x^{\prime T}, p_{3}^{\prime m}\right) \in X \times P_{3}^{m} \mapsto S \subset X \times P_{3}^{m}
$$

where $S$ is a set of solutions for pre-assigned $\left(x^{\prime T}, p_{3}^{\prime m}\right)$. From the same argument in the proof of Proposition 1, we know that $\Psi$ is an upper-hemi-continuous correspondence, whose image is clearly compact and convex. Consequently,

$$
\exists\left(x^{* T}, p_{3}^{* m}\right) \in X \times P_{3}^{m} \quad \text { s.t. } \quad\left(x^{* T}, p_{3}^{* m}\right) \in \Psi\left(x^{* T}, p_{3}^{* m}\right)=S^{*} .
$$

Thus,

$$
\left\{\begin{array}{c}
x^{*} \text { maximizes } \hat{\Theta}\left(\beta\left(x^{*}\right), p_{3}^{* m}\right) x \\
\text { s.t. } A\left(g, \alpha\left(x^{*}\right), \beta\left(x^{*}\right)\right) x \leq L \\
\text { and } \\
p^{* m} \text { minimizes } p^{m} L \\
\text { s.t. } p^{m} A\left(r, \alpha\left(x^{*}\right), \beta\left(x^{*}\right)\right) \geq \hat{\Theta}\left(\beta\left(x^{*}\right), p_{3}^{* m}\right),
\end{array}\right.
$$


which implies that $\left(x^{* T}, p^{* m}\right)$ is a solution. Non-triviality of an equilibrium solution is obvious.

\section{Appendix 2}

Proof of Proposition 3 Suppose there are positive lower bounds for $x_{3}^{*}$ and $x_{5}^{*}$ as $\theta_{i} \rightarrow \infty(i=4,5)$. Denote them as $\bar{x}_{3}$ and $\bar{x}_{5}$, respectively.

Consider a sequence $\left\{\theta_{v}\right\} \equiv\left\{\left(\theta_{4 v}, \theta_{5 v}\right)\right\}$ such that $\theta_{i v} \rightarrow \infty$ as $v \rightarrow \infty$. Since $X$ is a compact set, we can choose a sequence $\left\{x_{v}^{*}\right\}$ which is a solution corresponding to $\theta_{v}$ such that $x_{3 v}^{*} \rightarrow \bar{x}_{3}$ and $x_{5 v}^{*} \rightarrow \bar{x}_{5}$. Then, we have

$$
x_{2 v}^{*}+x_{3 v}^{*}-\left\{\theta_{4}\left(1-\psi_{v}^{*}\right) x_{4 v}^{*}+\theta_{5} x_{5 v}^{*}\right\} \rightarrow-\infty
$$

where $1-\psi_{v}^{*} \equiv x_{2 v}^{*} /\left(\phi_{2}\left(\beta_{v}^{*}\right) x_{2 v}^{*}+x_{3 v}^{*}\right)$ and $\psi_{v}^{*} \rightarrow \bar{\psi}<1$, since $\bar{x}_{3}^{*}$ is assumed to be positive.

This, however, contradicts that the above is the maximized value, which cannot be negative.

Proof of Proposition 4 From the second inequality of (7) and $p_{1}^{m *}=1$, we know that at least one of the $p_{1}^{m *}$ and $w^{m *}$ is positive. Let me show that both $p_{1}^{m *}$ and $w^{m *}$ are positive. If $w^{m *}>0=p_{1}^{m *}$ holds,

$$
(1+r) a_{1} p_{1}^{m *}+l_{1} w^{m *}>p_{1}^{m *}=0
$$

holds, so that $x_{1}^{m *}=0$ holds. But this contradicts non-triviality of a solution. If $0=w^{m *}<p_{1}^{m *}$ holds, we have

$$
0=l_{1} w^{m *} \geq\left\{1-(1+r) a_{1}\right\} p_{1}^{m *}>0 .
$$

A contradiction. Thus, both $p_{1}^{m *}$ and $w^{m *}$ are positive.

Then, from the second and third inequalities of (7), we have

$$
(1+r) a_{2}(\beta) p_{1}^{m *}+w^{m *} l_{2}(\beta)>(1+r) a_{3} p_{1}^{m *}+l_{3} w^{m *}
$$

due to Assumption 3, so that $x_{2}^{m *}=0$. Applying the same argument to the fourth and fifth inequalities of (7), we have $x_{4}^{m *}=0$.

Proof of Proposition 5 Look at the right-hand side of the fourth and fifth inequalities of (4). Since $\theta_{i}$ is assumed to be sufficiently small, we know that $x_{4}^{*}=0$ and $x_{5}^{*}>0$, so that $\beta^{*}=0$, due to Assumption 5 .

Since the first inequality must hold with strict equality, we have

$$
w^{*}=\frac{\left\{1-(1+r) a_{1}\right\} p_{1}^{* s}}{l_{1}} .
$$


Substitute this into the second and third inequalities of (4) and take $\beta^{*}=0$ into account.

Then, we have

$$
\begin{aligned}
& p_{1}^{s *}\left[(1+r) a_{2}(0)+\phi_{2}(0)\left[a_{5}+\frac{l_{5}}{l_{1}} \frac{\left\{1-(1+r) a_{1}\right\}}{1+r}-\theta_{5}\right]\right. \\
& \left.\quad+\frac{l_{2}(0)}{l_{1}}\left\{1-(1+r) a_{1}\right\}\right] \geq p_{2}^{s *}(=1) \\
& p_{1}^{s *}\left[(1+r) a_{3}+\left[a_{5}+\frac{l_{5}}{l_{1}} \frac{\left\{1-(1+r) a_{1}\right\}}{1+r}-\theta_{5}\right]\right. \\
& \left.\quad+\frac{l_{3}}{l_{1}}\left\{1-(1+r) a_{1}\right\}\right] \geq p_{2}^{s *}(=1),
\end{aligned}
$$

where a strict equality must hold in either inequality above. Hence, if the left-hand side of the first inequality above is smaller than that in the second, then the second production process is chosen for commodity production. If the inequality sign is the opposite, then the third process is chosen. In the former case, we have $x_{2}^{*}>0$ and $x_{3}^{*}=0$, while we have $x_{2}^{*}=0$ and $x_{3}^{*}>0$ in the latter case.

Considering $\theta_{5} \cong 0$, if

$$
1-\phi_{2}(0)>\frac{1+r}{l_{5}+(1+r)\left(a_{5} l_{1}-l_{5} a_{1}\right)}\left[(1+r) l_{1}\left\{a_{2}(0)-a_{3}\right\}+\left\{1-(1+r) a_{1}\right\}\left\{l_{2}(0)-l_{3}\right\}\right] .
$$

holds, the former case is valid. Clearly, this holds when both $l_{1}$ and $\left\{1-(1+r) a_{1}\right\}$ are sufficiently small, since the right-hand side is sufficiently small and close to zero. The same is true if both $\left|a_{2}(0)-a_{3}\right|$ and $\left|l_{2}(0)-l_{3}\right|$ are sufficiently small. Thus, the proposition follows.

\section{Appendix 3}

Proof of Proposition 8 Due to Proposition 5, we have

$$
\left\{\begin{aligned}
(1+g)\left\{a_{1} x_{1}^{*}+a_{2}(0) x_{2}^{*}+a_{5} x_{5}^{*}\right\} & =x_{1}^{*} \\
\phi_{2}(0) x_{2}^{*} & =(1+g) x_{5}^{*} \\
l_{1} x_{1}^{*} l_{2}(0) x_{2}^{*}+l_{5} x_{5}^{*} & =l
\end{aligned}\right.
$$

from which we can obtain the following:

$$
x_{2}^{*}=\frac{(1+g)\left\{1-(1+g) a_{1}\right\}}{\left\{(1+g) a_{2}(0)+a_{5} \phi_{2}(0)\right\} l_{1}+\left\{1-(1+g) a_{1}\right\}\left\{(1+g) l_{2}(0)+l_{5} \phi_{2}(0)\right\}} l .
$$

On the other hand, considering Proposition 4, we can calculate

$$
x_{3}^{m *}=\frac{(1+g)\left\{1-(1+g) a_{1}\right\}}{\left\{(1+g) a_{3}+a_{5}\right\} l_{1}+\left\{1-(1+g) a_{1}\right\}\left\{(1+g) l_{3}+l_{5}\right\}} l .
$$

Compare the above equations. Then, $x_{2}^{*}>x_{3}^{m *}$ holds if the following holds: 


$$
\begin{gathered}
\left\{\begin{array}{l}
(1+g) a_{2}(0)+\phi_{2}(0) a_{5}<(1+g) a_{3}+a_{5} \\
(1+g) l_{2}(0)+\phi_{2}(0) l_{5}<(1+g) l_{3}+l_{5} .
\end{array}\right. \\
\Leftrightarrow\left\{\begin{array}{l}
1-\phi_{2}(0)>\frac{(1+g)\left\{a_{2}(0)-a_{3}\right\}}{a_{5}} \\
1-\phi_{2}(0)>\frac{(1+g)\left\{l_{2}(0)-l_{3}\right\}}{l_{5}} .
\end{array}\right.
\end{gathered}
$$

Hence, if

$$
1-\phi_{2}(0)>(1+g) \max \left(\frac{a_{2}(0)-a_{3}}{a_{5}}, \frac{l_{2}(0)-l_{3}}{l_{5}}\right)
$$

holds, we have $x_{2}^{*}>x_{3}^{m *}$. Therefore, due to Proposition 5,

$$
\Theta\left(\psi^{*}\right) x^{*}=\Theta(1) x *=x_{2}^{*}-\theta_{5} x_{5}^{*}>x_{3}^{m *}-\theta_{5} x_{5}^{m *}=\Theta\left(\alpha^{m *}\right) x^{m *}
$$

if $\theta_{5}$ is sufficiently small.

\section{Appendix 4}

Proof of Proposition 9 Denote each production and disposal process as $k=I, I I \ldots, V$. Then, production techniques are expressed by $\{I, I I, I V\},\{I, I I, V\},\{I, I I I, I V\}$ and $\{I, I I I, V\}$, respectively, and they are labeled as $\{i\},\{i i\}, \ldots,\{i v\}$. Let me calculate the price of the consumption commodity of the sustainable equilibrium in terms of the wage rate, which corresponds to each technique.

First, it must be noted that $\theta_{4}$ and $\theta_{5}$ are coefficients of environmental burdens and independent of choice of technique. Furthermore, $p_{1}^{s} / w^{s}$ is also independent of choice of technique by construction, so that

$$
\left(\frac{p_{1}^{s}}{w^{s}}\right)^{\{i\}}=\cdots=\left(\frac{p_{1}^{s}}{w^{s}}\right)^{\{i v\}}=\frac{l_{1}}{1-(1+r) a_{1}}
$$

holds, where $z^{\{i\}}(k=i, \ldots, i v)$ shows a variable $z$ obtained in an economy of a $\{k\}$-technique. Hence, I express $\left(p_{1}^{s} / w^{s}\right)^{\{k\}}$ simply as $\left(p_{1}^{s} / w^{s}\right)$ for $k=1, \ldots, i v$.

Now, consider the production technique $\{i\}$ (that is, $\{I, I I, I V\}$ ), where the price of a consumption commodity in a sustainable equilibrium is denoted $p_{2}^{s\{i\}}$. It is easily calculated as

$$
\left(\frac{p_{2}^{s}}{w^{s}}\right)^{\{i\}} \equiv\left(\frac{1}{w^{s}}\right)^{\{i\}}=\left\{(1+r) a_{2}(1)+\phi_{2}(1) a_{4}(1)\right\} \frac{p_{1}^{s}}{w^{s}}+\left\{\frac{\phi_{2}(1)}{1+r} l_{4}(1)+l_{2}(1)\right\}
$$

In the same way, for $\{I, I I, V\},\{I, I I I, I V\}$ and $\{I, I I I, V\}$, we have

$$
\begin{aligned}
& \left(\frac{p_{2}^{s}}{w^{s}}\right)^{\{i i\}} \equiv\left(\frac{1}{w^{s}}\right)^{\{i i\}}=\left\{(1+r) a_{2}(0)+\phi_{2}(0) a_{5}\right\} \frac{p_{1}^{s}}{w^{s}}+\left\{\frac{\phi_{2}(0)}{1+r} l_{5}+l_{2}(0)\right\}+\frac{\phi_{2}(0)}{1+r}\left(\frac{\theta_{5}}{w^{s}}\right)^{\{i i\}}, \\
& \left(\frac{p_{2}^{s}}{w^{s}}\right)^{\{i i i\}} \equiv\left(\frac{1}{w^{s}}\right)^{\{i i i\}}=\left\{(1+r) a_{3}+a_{4}(0)\right\} \frac{p_{1}^{s}}{w^{s}}+\left\{\frac{1}{1+r} l_{4}(0)+l_{3}\right\}+\frac{1}{1+r}\left(\frac{\theta_{4}}{w^{s}}\right)^{\{i i i\}},
\end{aligned}
$$


and

$$
\left.\left(\frac{p_{2}^{s}}{w^{s}}\right)^{\{i v\}} \equiv\left(\frac{1}{w^{s}}\right)^{\{i v\}}=\left\{(1+r) a_{3}+a_{5}\right)\right\} \frac{p_{1}^{s}}{w^{s}}+\left\{\frac{1}{1+r} l_{5}+l_{3}\right\}+\frac{1}{1+r}\left(\frac{\theta_{5}}{w^{s}}\right)^{\{i v\}}
$$

From the above calculation, we have

$$
\begin{aligned}
\left(\frac{p_{1}^{s}}{w^{s}}\right)^{\{i i\}}-\left(\frac{p_{1}^{s}}{w^{s}}\right)^{\{i\}} & =\left[(1+r)\left\{a_{2}(0)-a_{2}(1)\right\}+\left\{\phi_{2}(0) a_{5}-\phi_{2}(1) a_{4}(1)\right\}\right] \frac{p_{1}^{s}}{w^{s}} \\
+ & {\left[\frac{1}{1+r}\left\{\phi_{2}(0) l_{5}-\phi_{2}(1) l_{4}(1)\right\}+\left\{l_{2}(0)-l_{2}(1)\right\}\right]+\frac{\phi_{2}(0)}{1+r}\left(\frac{\theta_{5}}{w^{s}}\right)^{\{i i\}} } \\
\left(\frac{p_{1}^{s}}{w^{s}}\right)^{\{i i i\}}-\left(\frac{p_{1}^{s}}{w^{s}}\right)^{\{i\}}= & {\left[(1+r)\left\{a_{3}-a_{2}(1)\right\}+\left\{a_{4}(0)-\phi_{2}(1) a_{4}(1)\right\}\right] \frac{p_{1}^{s}}{w^{s}} } \\
+ & {\left[\frac{1}{1+r}\left\{l_{4}(0)-\phi_{2}(1) l_{4}(1)\right\}+\left\{l_{3}-l_{2}(1)\right\}\right]+\frac{1}{1+r}\left(\frac{\theta_{4}}{w^{s}}\right)^{\{i i i\}} } \\
\left(\frac{p_{1}^{s}}{w^{s}}\right)^{\{i v\}}-\left(\frac{p_{1}^{s}}{w^{s}}\right)^{\{i\}}= & {\left[(1+r)\left\{a_{3}-a_{2}(1)\right\}+\left\{a_{5}-\phi_{2}(1) a_{4}(1)\right\}\right] \frac{p_{1}^{s}}{w^{s}} } \\
+ & {\left[\frac{1}{1+r}\left\{l_{5}-\phi_{2}(1) l_{4}(1)\right\}+\left\{l_{3}-l_{2}(1)\right\}\right]+\frac{1}{1+r}\left(\frac{\theta_{5}}{w^{s}}\right)^{\{i v\}} . }
\end{aligned}
$$

Therefore, there exists sufficiently small positive $\varepsilon$ such that, if $\left|a_{j}-a_{i}(0)\right|<\varepsilon$ and $\left|l_{j}-l_{i}(0)\right|<\varepsilon$ hold, then $\left(1 / w^{s}\right)^{\{k\}}>\left(1 / w^{s}\right)^{\{i\}}$ holds for $k=i i, i i i, i v$, where $(i, j)=(2,3)$ and $(4,5)$. Clearly, if $\theta_{i}(i=, 4,5)$ is sufficiently large, $\left(1 / w^{s}\right)^{\{k\}}>\left(1 / w^{s}\right)^{\{i\}}$ also holds for $k=i i, i i i, i v$.

Then, the techniques $\{i i\},\{i i i\}$ and $\{i v\}$ are less profitable than the technique $\{i\}$, so that the former techniques are not adopted. Yet, it might be considered that a certain combination of those techniques could be equi-profitable. Such a situation never occurs, since the above procedure is still valid with minor modifications such as $0<\alpha<1,0<\beta<0$ and $0<\psi<1$.

Moreover, if $\left|a_{j}-a_{i}(0)\right|<\varepsilon$ and $\left|l_{j}-l_{i}(0)\right|<\varepsilon$ hold, clearly $\left|a_{j}-a_{i}(h)\right|<\varepsilon$ and $\left|l_{j}-l_{i}(h)\right|<\varepsilon$ hold for $(i, j, h)=(2,3, \beta)$ and $(4,5, \alpha)$. Thus, I have shown the following lemma:

Lemma 1 (i) If $\theta_{i}(i=4,5)$ is sufficiently large or (ii) if $\left|a_{j}-a_{i}(0)\right|<\varepsilon$ and $\left|l_{j}-l_{i}(0)\right|<\varepsilon$ holds for sufficiently small $\varepsilon$, where $(i, j)=(2,3)$ and $(4,5)$, respectively, then $\left(p_{2}^{s} / w^{s}\right)^{\{k\}}=\left(1 / w^{s}\right)^{\{k\}}>\left(1 / w^{s}\right)^{\{i\}}=\left(p_{2}^{s} / w^{s}\right)^{\{i\}}$ for $k=i i, i i i$, iv holds.

Thus, the production technique $\{i\}$ (that is, $\{I, I I, I V\}$ ) is chosen in a sustainable equilibrium.

Next, let me consider the Lagrangean of (13) as follows:

$$
\mathcal{L} \equiv \Theta(\psi(\beta(x)))-\lambda\{A(g, \alpha(x), \beta(x)) x-L\}
$$

where $\lambda$ is a Lagrangean vector $\lambda \equiv\left(\lambda_{1}, \lambda_{2}, \lambda_{3}\right)$. Then, from the Kuhn-Tucker conditions

$$
\frac{\partial \mathcal{L}_{i}}{\partial x_{i}} \leq 0 \text { and } \frac{\partial \mathcal{L}_{i}}{\partial x_{i}} x_{i}=0
$$


must hold for $i=1, \ldots, 5$.

$$
\begin{aligned}
& \mathcal{L}_{1}=\lambda_{1}\left\{1-(1+g) a_{1}\right\}-\lambda_{3} l_{1} \leq 0 \\
& \mathcal{L}_{2}=1+\frac{\phi_{2}(\beta) \theta_{4} x_{3} x_{4}}{\left\{\phi_{2}(\beta) x_{2}+x_{3}\right\}^{2}}-\lambda_{1}(1+g)\left\{a_{2}(\beta)+\frac{a_{4}^{\prime}(\alpha) x_{3} x_{4}}{\left(x_{2}+x_{3}\right)^{2}}\right\}-\lambda_{2} \phi_{2}(\beta) \\
& -\lambda_{3}\left\{l_{2}(\beta)+\frac{l_{4}^{\prime}(\alpha) x_{3} x_{4}}{\left(x_{2}+x_{3}\right)^{2}}\right\} \leq 0 \\
& \mathcal{L}_{3}=1-\frac{\phi_{2}(\beta) \theta_{4} x_{2} x_{4}}{\left\{\phi_{2}(\beta) x_{2}+x_{3}\right\}^{2}}-\lambda_{1}(1+g)\left\{a_{3}-\frac{a_{4}^{\prime}(\alpha) x_{2} x_{4}}{\left(x_{2}+x_{3}\right)^{2}}\right\}-\lambda_{2} \\
& -\lambda_{3}\left\{l_{3}-\frac{l_{4}^{\prime}(\alpha) x_{2} x_{4}}{\left(x_{2}+x_{3}\right)^{2}}\right\} \leq 0 \\
& \mathcal{L}_{4}=-\theta_{4}(1-\psi)-\frac{\phi_{2}^{\prime}(\beta) \theta_{4} x_{2} x_{3} x_{4}}{\left\{\phi_{2}(\beta) x_{2}+x_{3}\right\}^{2}} \frac{x_{5}}{\left(x_{4}+x_{5}\right)^{2}}-\lambda_{1}(1+g)\left\{\frac{a_{2}^{\prime}(\beta) x_{2} x_{5}}{\left(x_{4}+x_{5}\right)^{2}}+a_{4}(\alpha)\right\} \\
& -\lambda_{2}\left\{\frac{\phi_{2}^{\prime}(\beta) x_{2} x_{5}}{\left(x_{4}+x_{5}\right)^{2}}-(1+g)\right\}-\lambda_{3}\left\{\frac{l_{2}^{\prime}(\beta) x_{2} x_{5}}{\left(x_{4}+x_{5}\right)^{2}}+l_{4}(\alpha)\right\} \leq 0 \\
& \mathcal{L}_{5}=-\frac{\phi_{2}^{\prime}(\beta) \theta_{4} x_{2} x_{3} x_{4}^{2}}{\left\{\phi_{2}(\beta) x_{2}+x_{3}\right\}^{2}\left(x_{4}+x_{5}\right)^{2}}-\lambda_{1}(1+g)\left\{-\frac{a_{2}^{\prime}(\beta) x_{2} x_{4}}{\left(x_{4}+x_{5}\right)^{2}}+a_{5}\right\} \\
& -\lambda_{2}\left\{-\frac{\phi_{2}^{\prime}(\beta) x_{2} x_{4}}{\left(x_{4}+x_{5}\right)^{2}}-(1+g)\right\}-\lambda_{3}\left\{-\frac{l_{2}^{\prime}(\beta) x_{2} x_{4}}{\left(x_{4}+x_{5}\right)^{2}}+l_{5}\right\} \leq 0 \text {. }
\end{aligned}
$$

Thus, we have

$$
\begin{aligned}
\mathcal{L}_{2}-\mathcal{L}_{3}= & 1+\frac{\theta_{4} \phi_{2}(\beta) x_{4}\left(x_{2}+x_{3}\right)}{\left\{\phi_{2}(\beta) x_{2}+x_{3}\right\}^{2}}-\lambda_{1}(1+g)\left[\left\{a_{2}(\beta)-a_{3}\right\}+\frac{l_{4}^{\prime}(\alpha) x_{4}}{x_{2}+x_{3}}\right] \\
- & \lambda_{2}\left\{\phi_{2}(\beta)-1\right\}-\lambda_{3}\left[\left\{l_{2}(\beta)-l_{3}\right\}+\frac{l_{4}^{\prime}(\alpha) x_{4}}{x_{2}+x_{3}}\right] \\
\mathcal{L}_{4}-\mathcal{L}_{5}= & -\theta_{4}(1-\psi)+\theta_{5}+\frac{\phi_{2}^{\prime}(\beta) \theta_{4} x_{2} x_{3}}{\left\{\phi_{2}(\beta) x_{2}+x_{3}\right\}^{2}\left(x_{4}+x_{5}\right)} \\
& -\lambda_{1}(1+g)\left[\frac{a_{2}^{\prime}(\beta) x_{2}}{x_{4}+x_{5}}+\left\{a_{4}(\alpha)-a_{5}\right\}\right]-\lambda_{2} \frac{\phi_{2}^{\prime}(\beta) x_{2}}{x_{4}+x_{5}} \\
& -\lambda_{3}\left[\frac{l_{2}^{\prime}(\beta) x_{2}}{x_{4}+x_{5}}+\left\{l_{4}(\alpha)-l_{5}\right\}\right] .
\end{aligned}
$$

Hence, if $\left|a_{2}(\beta)-a_{3}\right|<\epsilon_{1}$ and $\left|l_{2}(\beta)-l_{3}\right|<\epsilon_{1}$ for sufficiently small $\epsilon_{1}$ and for all $(\alpha, \beta) \in[0,1] \times[0,1]$, then $\mathcal{L}_{2}>\mathcal{L}_{3}$ holds, which implies $x_{2}>0, x_{3}=0$ and $\psi=1$. Thus, the first and third terms of $\mathcal{L}_{4}-\mathcal{L}_{5}$ equal zero. Consequently, if $\left|a_{4}(\alpha)-a_{5}\right|<\epsilon_{2}$ and $\left|l_{4}(\alpha)-l_{5}\right|<\epsilon_{2}$ for sufficiently small $\epsilon_{2}$ and for all $(\alpha, \beta) \in[0,1] \times[0,1]$, then $\mathcal{L}_{4}>\mathcal{L}_{5}$ holds. The same argument follows when $\theta_{i}(i=1,2)$ is sufficiently large. Therefore, we have the following result:

Lemma 2 (i) If $\theta_{i}(i=4,5)$ is sufficiently large or (ii) if $\left|a_{j}-a_{i}(0)\right|<\epsilon$ and $\left|l_{j}-l_{i}(0)\right|<\epsilon$ holds for sufficiently small $\epsilon$, where $(i, j)=(2,3)$ and $(4,5)$, respectively, then $x_{3}^{* *}=0$ and $x_{5}^{* *}=0$.

From Lemmas 1 and 2, Proposition 9 follows. 
Received: 9 July 2015 Accepted: 3 February 2016

Published online: 03 March 2016

\section{References}

ACEA (2011) ACEA position on dismantling of end-of-life vehicles by non-authorized treatment operators. European Automobile Manufacturer's Association

European Commission (2014) European resource efficiency platform. Manifesto and Policy Recommendation, European Commission

Franke R (1986) Some problems concerning the notion of cost-minimizing systems in the framework of joint production. Manch Sch LIV(3):298-307

Fujimoto T, Opocher A (2010) Commodity content in a general input-output model. Metroeconomica 61(3):442-453

Gale D (1960) The theory of linear economic models. McGraw-Hill, New York

Gehrke C, Lager C (1995) Environmental taxes, relative prices and choice of technique in a linear model of production. Metroeconomica 46(2):127-145

Hosoda E (2010a) Malfunction of a market in a transaction of waste: a reason for the necessity of an upstream policy in waste management. In: Vint J (ed) Economic theory and economic thought-essays in honour of lan Steedman. Routledge, London, pp 234-253

Hosoda E (2010b) Bads as joint products in a linear production system. In: Birolo A et al (eds) Production, distribution and trade. Routledge, London, pp 33-61

Hosoda E (2012) Recycling of waste and downgrading of secondary resources in a classical type of production model. $J$ Econ Struct 1:7. doi:10.1186/2193-2409-1-7

Hosoda E (2014) Sorting and recycling in a multi-sectoral economy: a neo-Ricardian approach. Metroeconomica 65(1):58-94. doi:10.1111/meca.12029

Kurz HD (2008) Innovations and profits Schumpeter and the classical heritage. J Econ Behav Organ 67:263-278

Kurz HD, Salvadori N (1995) Theory of production: a long-period-analysis. Cambridge University Press, Cambridge

Lager C (1999) Perverse results of a greening of the tax system. In: Teixeira JR, Carneiro FG (eds) Economic dynamics and economic policy. Brasilia, DF, pp 147-160

Lager C (2001) Joint production with restricted free disposal. Metroeconomica 52(1):49-78

Sraffa P (1960) Production of commodities by means of commodities. Cambridge University Press, Cambridge

Steedman I (1975) Positive profits with negative surplus value. Econ J 85:604-608

Steedman I (1977) Marx after sraffa. NLB, London

Ueno K (2004) Recycling and eco-design of household electric appliances. Waste Manag Res 15(3):115-122 (in Japanese)

\section{Submit your manuscript to a SpringerOpen ${ }^{\circ}$ journal and benefit from:}

Convenient online submission

Rigorous peer review

- Immediate publication on acceptance

- Open access: articles freely available online

- High visibility within the field

- Retaining the copyright to your article

Submit your next manuscript at $\$$ springeropen.com 